\title{
A Kuril Ainu Glossary by Captain V. M. Golovnin (1811)
}

\author{
Anna Bugaeva \\ Tokyo University of Science/NINJAL \\ Tokyo, Japan \\ bugaeva@rs.tus.ac.jp \\ Tomomi Satō \\ Hokkaido University \\ Sapporo, Japan \\ tomomis@let.hokudai.ac.jp
}

\begin{abstract}
This paper presents a newly discovered glossary (230 items) of Northern and Southern Kuril Ainu recorded by the captain Vasily Mikhaylovich Golovnin in 1811 and stored at the Russian National Archive of the Navy in St. Petersburg. Based on this new document we argue that Southern Kuril has a much closer lexical resemblance to Northeastern Hokkaido Ainu than Northern Kuril. On the other hand, we reaffirm that both Southern and Northern Kuril Ainu constitute a really separate Kuril group because they show a number of lexical, phonological and grammatical features, which are different from Hokkaido Ainu.
\end{abstract}

\section{Keywords}

Northern Kuril Ainu - Southern Kuril Ainu - Hokkaido Ainu - Vasily Mikhaylovich Golovnin - glossary - dialectology - philology 
Ainu is a nearly extinct Northeast Asian isolate formerly spoken in Hokkaido, ${ }^{1}$ Sakhalin, and the Kuril Islands. Kuril Ainu is the least documented variety of the language but it is absolutely indispensable for the history of Ainu. It disappeared in the beginning of the 2oth century as a result of constant territorial disputes over the Kuril Islands between Russia and Japan. According to the Treaty of Saint Petersburg (1875), the entire Kuril archipelago became Japanese territory and the whole Sakhalin Island became Russian. By that time, a hundred or so of the native Kurils (or the Kuril Ainu) who lived on the former Russian part of the Kurils had been already considerably Russified and baptized (Zajac 2009: 34-52). In order to assimilate the Kuril Ainu, who were perceived of as a potential Russian threat by the Japanese, the Japanese government forcedly moved most of them to the southern island of Shikotan in 1884. The living conditions in Shikotan were different and also rather poor compared to the other Kuril Islands, which led to the death of many Kuril Ainu people and the eventual loss of their language (Zajac 20o9: 99-101).

There is only less than a dozen of published Kuril Ainu documents containing word lists and short phrases, i.e. about 297 items in Krasheninnikov (1755), 372 items in Klaproth (1823), 1900 items in Dybowski (1892), 700 items in Torii (1903), 30 items by Argunov (Satō and Bugaeva 2019, Bugaeva and Gorlova 2021) and 500 out of 1600 items from Voznesensky's archive (SPbF-ARAN, f. 53, op.1, \#43) published in Vovin (1993), for a fuller description see Alonso de la Fuente (2021: $3-7)$. All these materials present a Northern Kuril variety of Ainu while Southern Kuril Ainu remains undocumented.

This article is the first attempt to discuss the differences between Northern and Southern Kuril Ainu based on a newly discovered glossary from the captain Vasily Mikhaylovich Golovnin's archive stored at the Russian National Archive of the Navy in St. Petersburg (RGAVMF, f. 7, op. 1, \#19, pp. 199-203). The document contains 230 items with forms in both Northern Kuril (called язык русских курильцев 'the language of the Russian Kurils') and Southern Kuril Ainu (called язык мохнатых 'the language of the hairy people'). ${ }^{2}$ It was written by the captain V. M. Golovnin himself during his 1811 trip aimed

1 Hokkaido Ainu dialects are divided into Southwestern and Northeastern groups.

2 The Russians called the Southern Kuril Ainu and Hokkaido Ainu мохнатые 'hairy people' probably because of their beards. The Northern Kuril Ainu who lived on the Russian territory adopted a Russian custom of shaving beards so they were not called like that (Golovnin 1816: 220-221). 
at describing the Kuril Islands. ${ }^{3}$ This study proves that the Kuril Ainu group of dialects should be divided into Northern and Southern subgroups because there are certain differences in the lexicon and grammar, which, do not, however hinder mutual comprehensibility.

The article has the following structure. First, we explain the details of V. M. Golovnin's trip (1811) to Kurils and try to make an assumption about the place, time and circumstances in which the glossary was compiled (§2). Next, we characterize the glossary and its accompanying narrative with short phrases and present them $\left(\S_{3}\right)$. And finally, we discuss the linguistic characteristics of Kuril Ainu presented in the document $\left(\S_{4}\right)$ and turn to concluding remarks $(\$ 5)$.

\section{M. Golovnin and His 1811 Trip to Kurils}

Vasily Mikhaylovich Golovnin (1776-1831) is a Russian Navy officer and seafarer. In 1806, Golovnin was given command of the sloop Diana and made his first trip around the world (1807-1809) to survey the northern Pacific. At that time, there was no official border ${ }^{4}$ between Russia and Japan but Russia had control over about sixteen Kuril Islands counting from the North, while the Southern Kuril islands of Iturup and Kunashir were inhabited by the Japanese (and the Ainu). In the end of April 1811, the captain Golovnin sailed off Kamchatka being commissioned to chart the coasts of the Southern Kuril Islands. Golovnin had managed to survey at least seven Kuril Islands, i.e. Rasshua (\#13), Ushishir (\#14), Ketoy (\#15), Simushir (\#16), two Chirpoys (\#17), and Urup (\#18), before his six crew members and him, as well as the Kuril Ainu Alexey were taken prisoners by the Japanese on Kunashir on July 11, 1811 and spent two years in captivity in Hakodate and Matsumae of Hokkaido (Golovnin 1816: 204).

The question is where and under what circumstances he could have written down the present glossary? This would hardly be possible in captivity because at most times Golovnin had no paper (Golovnin 2007:273) and almost no freedom to do what he wanted. Even though he was often accompanied by Kumajirō Uehara, a famous Japanese-Ainu interpreter and the author of

3 Olga Kanamatovna Gerbekova and other specialists from the Ryazan' Regional Academic Library, the compilers of a full catalogue of V. M. Golovnin's library (Ministry of culture and tourism of Ryazan' Region, the Ryazan' Regional Academic Library 2021), helped us identify V. M. Golovnin's handwriting with a great degree of accuracy. It should be noted that Vasily Mikhaylovich Golovnin was born in the Ryazan' Region.

4 In 1855, Russia and Japan concluded the Treaty of Commerce, Navigation and Delimitation and established the official border between Iturup and Urup Islands. 
the first Ainu dictionary Moshiogusa (もしほ草) 'Seaweeds for making salt' (1792) (Narita and Kindaichi 1972), it is very unlikely that the latter would teach Golovnin Ainu; it was rather Golovnin's job to teach Uehara Russian. In 1813, Golovnin and other sailors were finally released from Japanese captivity by the captain Pëtr Ivanovich Ricord (1776-1855) who exchanged them for several Japanese merchants abducted by Khvostov and Davydov in 1807.5

The only possibility to compile the glossary would be just a few weeks before the captivity. In fact, there is a detailed account of Golovnin's survey of the Kuril Islands, which includes all of his crewmen's encounters with the local Ainu, ${ }^{6}$ namely in Ketoy, Rasshua, and twice in Ushishir (Golovnin 1819: $16,33,36,38)$. However, those accounts do not mention any documentation of Kuril Ainu; most conversations with the Ainu were carried out in Russian. On June 19, Diana was near Iturup Island (Golovnin 1819: 79) and Golovnin ordered two of his crewmen to inspect the island where they met some Japanese people, local Kuril Ainu and nine Kuril Ainu from the Russian Island Rasshua (\#13). The Ainu from Rasshua had shipwrecked ${ }^{7}$ off the coast of Iturup a year ago and had been taken prisoners by the Japanese as Russian spies because of the 1807 year's incident (see footnote 5 ). However, by the time Diana arrived, they were released and were making preparations to sail back to Rasshua. They visited Diana and one of them, a young man Alexey, who was most proficient in Russian, was invited to sail together on Diana to help survey Urup and Kunashir Islands. That was exactly the time when Golovnin could have recorded the glossary from Alexey who was a native speaker of Northern Kuril Ainu (Rasshua) and knew Southern Kuril Ainu ${ }^{8}$ as well after one year spent on Iturup.

In captivity Alexey served as a translator from Kuril Ainu into Russian while the above-mentioned official Japanese translator Kumajirō Uehara served as a translator from Japanese into Kuril Ainu; the Ainu language was used as a lingua-franca by the imprisoned captain Golovnin and the Japanese.

5 "In 1807, without authorization (and seemingly counterproductive to the goal of opening peaceful trade), Rezanov ordered naval officers (also employees of the Russian-American Company) Nikolai A. Khvostov and G. I. Davydov to attack Japanese possessions in the North Pacific. They attacked fishery outposts on southern Sakhalin, Rishiri Island, and defeated Nanbu and Tsugaru garrisons on Iturup (Etorofu) Island and took several Japanese prisoners." (Walker 2007: 298).

6 According to Golovnin (1819: 119), there were only about 15o Ainu people on all Russian Kurils but much more of them on the four Japanese Southern Kuril Islands.

7 According to the Ainu themselves, they came to Irup in order to trade with the Japanese (Golovnin 1816: 216).

8 Golovnin complained that once he was on the boat, every time he tried to speak to the Japanese, Alexey was constantly chatting with the Iturup Ainu (Golovnin 1816: 227-228). 
Golovnin (1811) consists of two parts, i.e. Словарь Курильского языка [A Kuril Ainu Glossary] and a narrative about Kuril Ainu, which follows the glossary. The glossary contains 201 Russian glosses including those for which there are no Kuril Ainu words, see Table 1 below. We also present a full text of the narrative in Russian in the pre-reform Russian orthography and our English translation of it in Table 2. The narrative includes 29 Ainu words/phrases for a total of 230 items.

The glossary (Table 1) has three columns: a Russian gloss written in the prereform Russian orthography (column 1), a Northern Kuril Ainu form (column 3), and a Southern Kuril Ainu form (column 5) with a focus on Southern Kuril Ainu: when the Northern Kuril Ainu form is the same it is not repeated. Ainu forms are recorded in the Cyrillic script in the pre-reform Russian orthography but some letters contain the Latin letters above them to render a closer phonetic representation; those upper Latin letters are reflected here at the upper right corner of the Russian letter they refer to, e.g. $\mathrm{x}^{\mathbf{h}}, \mathrm{\Gamma}^{\mathbf{h}}, \mathrm{u}^{\mathbf{h}} ; \mathrm{T}^{\text {th }}, \mathrm{T}^{\text {th }} \mathrm{u}, \mathrm{T}^{\text {th }} \mathrm{p} ; \mathrm{B}^{\mathbf{w}}$ (Russian letters: $\mathrm{x}[\mathrm{h}], \mathrm{r}[\mathrm{g}]$, ч $[\mathrm{t}]$ ], т $[\mathrm{t}], \mathrm{p}[\mathrm{r}]$, в $[\mathrm{w}]$ ).

Moreover, we have added to the glossary three extra columns, i.e. column 2 with an English translation of the Russian gloss and columns 4 and 6 for our interpretation of Northern and Southern Kuril Ainu forms respectively. Both interpretation columns, they are in italics, contain the morphemic divisions of words and morphemic glossing in \{\} . Many interpretations require an explanation, which is consistently provided in footnotes.

It is not indicated whether the forms in the narrative are from Northern or Southern Kuril Ainu but we suggest that they are from Northern (Rasshua) Ainu, which is the native dialect of the speaker Alexey. We mark the original Ainu words in the narrative both in the Cyrillic and Latin alphabets in bold and our added Ainu interpretations are in italics.

The order of words in the glossary is thematic (the numbering is ours):

1. -36 . Natural phenomena, the four elements, seasons, and geographical features;

37.-49. Names for people and kinship terms;

50.-67. Body parts;

68.-89. Animals;

90.-94. Clothes;

95.-99. Food and drinks;

100.-119. Tools and utensils;

120.-122. Color terms; 
123. Illness;

124.-129. Pronouns;

130.-157. Human actions and states;

158.-165. Cardinal and intercardial directions;

166. -201. Numerals.

The words/phrases in the narrative are less thematically structured but they include at least the following groups (numbering is ours):

202.-204. Imperative phrases (human actions);

205. Year;

206.-214. Numerals and numerals in combination with nouns;

215.-220. Names for weather and stars,

221.-222. Scolding and swearing;

223.-227. Ethnonyms;

228. What is this?

229.-230. Horse.

TABLE 1 Словарь Курильского языка ["A Kuril Ainu Glossary"]

\begin{tabular}{|c|c|c|c|c|}
\hline $\begin{array}{l}\text { (Russian } \\
\text { gloss) }\end{array}$ & $\begin{array}{l}\text { English } \\
\text { translation }\end{array}$ & $\begin{array}{l}\text { русских } \\
\text { курильцев } \\
\text { '(language) of } \\
\text { the Russian } \\
\text { Kurils' in } \\
\text { Cyrillic } \\
\text { = Northern Kuril } \\
\text { Ainu }\end{array}$ & $\begin{array}{l}\text { Interpretation } \\
1\end{array}$ & $\begin{array}{l}\text { мохнатых Interpretation } \\
\text { '(language) of the } \\
\text { hairy people' } \\
\text { in Cyrillic } \\
\text { = Southern Kuril } \\
\text { Ainu }\end{array}$ \\
\hline
\end{tabular}

\begin{tabular}{llll}
\hline 1. небо & sky & канто & kanto \\
2. солнце & sun & тчюпъ & cup \\
3. луна & moon & сиргуни & $\begin{array}{l}\text { sir-kunne cup } \\
\text { \{appearance- } \\
\text { black moon }\end{array}$ \\
\hline 4. облако & cloud & тчюпъ & nis \\
5. громъ & thunder & нисшъ & kamuy hum \\
& & камих ${ }^{\mathrm{h}}$ юмъ & god sound\} \\
6. молнія & lightning & & imeru
\end{tabular}

a In the table, only columns 1,3 , and 5 are original.

$\mathrm{b}$ This title is added.

c A loanword from Old Japanese kamï 'god', see \$4.2. 
TABLE 1 Словарь Курильского языка [“A Kuril Ainu Glossary”] (cont.)

\begin{tabular}{|c|c|c|c|c|c|}
\hline $\begin{array}{l}\text { (Russian } \\
\text { gloss) }\end{array}$ & $\begin{array}{l}\text { English } \\
\text { translation }\end{array}$ & $\begin{array}{l}\text { русских } \\
\text { курильцев } \\
\text { '(language) of } \\
\text { the Russian } \\
\text { Kurils' in } \\
\text { Cyrillic } \\
\text { = Northern } \\
\text { Kuril Ainu }\end{array}$ & Interpretation & $\begin{array}{l}\text { мохнатых } \\
\text { '(language) } \\
\text { of the hairy } \\
\text { people' } \\
\text { in Cyrillic } \\
\text { = Southern } \\
\text { Kuril Ainu }\end{array}$ & Interpretation \\
\hline
\end{tabular}

\begin{tabular}{|c|c|c|c|c|c|}
\hline 7. звьзда & star & & & кета & keta \\
\hline 8. вЂтеръ & wind & & & $\mathrm{T}^{\mathrm{th}} \mathrm{epa}$ & rera \\
\hline 9. туманъ & mist & & & урраръ & urar \\
\hline 10. снЊгъ & snow & & & убасъ & upas \\
\hline 11. дождь & rain & в ${ }^{\mathrm{w}}$ ини & $\begin{array}{l}\text { wen_i } \\
\{\text { bad thing }\}\end{array}$ & сирив ${ }^{\mathrm{w}}$ иль & $\begin{array}{l}\text { sir-wen } \\
\text { \{appearance- } \\
\text { bad }\}\end{array}$ \\
\hline 12. день & day & & & сирбигиръ & $\begin{array}{l}\text { sir-peker } \\
\text { \{appearance- } \\
\text { bright\} }\end{array}$ \\
\hline 13. НОчь & night & & & сиргуни & $\begin{array}{l}\text { sir-kunne } \\
\text { \{appearance- } \\
\text { black\} }\end{array}$ \\
\hline 14. утро & morning & & & нисабъ & nisat \\
\hline 15. вечеръ & evening & & & оннумакъ & onuman \\
\hline 16. полдень & noon & & & тоннуски & $\begin{array}{l}\text { to-noski } \\
\{\text { day-middle }\}\end{array}$ \\
\hline 17. весна & spring & & & пайгаръ & paykar \\
\hline 18. льто & summer & & & сакъ & sak \\
\hline 19. осень & autumn & & & тчюпъ & cup \\
\hline 2о. зима & winter & & & мата & mata \\
\hline 21. вода & water & ппе & pe & вакка & wakka \\
\hline 22. земля & earth & & & & \\
\hline 23. огонь & fire & & & аббе & ape \\
\hline 24. воздухъ & air & $\begin{array}{l}\text { слова ньть } \\
\text { 'no word' }\end{array}$ & & & \\
\hline
\end{tabular}


TABLE 1 Словарь Курильского языка [“A Kuril Ainu Glossary”] (cont.)

\begin{tabular}{|c|c|c|c|c|c|}
\hline $\begin{array}{l}\text { (Russian } \\
\text { gloss) }\end{array}$ & $\begin{array}{l}\text { English } \\
\text { translation }\end{array}$ & $\begin{array}{l}\text { русских } \\
\text { курильцев } \\
\text { '(language) of } \\
\text { the Russian } \\
\text { Kurils' in } \\
\text { Cyrillic } \\
\text { = Northern } \\
\text { Kuril Ainu }\end{array}$ & Interpretation & $\begin{array}{l}\text { мохнатых } \\
\text { '(language) } \\
\text { of the hairy } \\
\text { people' } \\
\text { in Cyrillic } \\
\text { = Southern } \\
\text { Kuril Ainu }\end{array}$ & Interpretation \\
\hline 25. материкъ & continent & & & $\begin{array}{l}\text { ирсине- } \\
\text { котанъ }\end{array}$ & $\begin{array}{l}\text { irsinne }^{\mathrm{d}} \text {-kotan } \\
\text { \{jointly-village }\}\end{array}$ \\
\hline 26. островъ & island & котанъ & kotan & мусиръ & mosir \\
\hline 27. море & sea & & & $\begin{array}{l}\text { атвига или } \\
\text { ‘or’ атуига }\end{array}$ & $\begin{array}{l}\text { atuy-ka } \\
\{\text { sea-surface }\}\end{array}$ \\
\hline 28. мысъ & cape & & & шеремгу & $\begin{array}{l}\text { sir-emko } \\
\{\text { land-end }\}\end{array}$ \\
\hline 29. сопка & volcanic hill & & & нубури & nupuri \\
\hline 3о. гора & mountain & & & $\Gamma^{\mathrm{h}}$ уръ & hur \\
\hline 31. озеро & lake & & & TO & to \\
\hline 32. гавань & harbor & & & тчибайни & $\begin{array}{l}\text { cip-e-yan-if } \\
\text { \{boat-at. } \\
\text { APPL-land- } \\
\text { place\} }\end{array}$ \\
\hline 33. проливъ & strait & & & атуй & $\begin{array}{l}\text { atuy } \\
\{\text { sea/ocean }\}\end{array}$ \\
\hline 34. рђка & river & & & петть & pet \\
\hline 35. сулой & tide-rip & & & тчу & $\operatorname{ciw}$ \\
\hline 36. заливъ & bay & & & мойнугоръ & $\begin{array}{l}\text { moy-nikor } \\
\text { \{whirlpool- } \\
\text { inside\} }\end{array}$ \\
\hline 37. человекъ & human & & & айну & аупи \\
\hline 38. мужчина & man & окаяйну & $\begin{array}{l}\text { okkay aynu } \\
\{\text { man human }\}\end{array}$ & окай & okkay \\
\hline
\end{tabular}

d Cf. irsine 'simultaneously, jointly' for Obihiro (Hokkaido) in Hattori (1964: 255) and issinne 'together' in Batchelor (1938: 207) and Kubodera (1992: 109), the latter has a note that the form is used in Sakhalin Ainu.

e In Kuril Ainu, kotan 'village' also means 'island, land' (Dybowski 1892: 42), see \#26.

f Also appears in Klaproth (1823: 307) and Voznesensky (1844: f. 53, op. 1, \#43, word no. 157). 
TABLE 1 Словарь Курильского языка [“A Kuril Ainu Glossary”] (cont.)

\begin{tabular}{|c|c|c|c|c|c|}
\hline $\begin{array}{l}\text { (Russian } \\
\text { gloss) }\end{array}$ & $\begin{array}{l}\text { English } \\
\text { translation }\end{array}$ & $\begin{array}{l}\text { русских } \\
\text { курильцев } \\
\text { '(language) of } \\
\text { the Russian } \\
\text { Kurils' in } \\
\text { Cyrillic } \\
\text { = Northern } \\
\text { Kuril Ainu }\end{array}$ & Interpretation & $\begin{array}{l}\text { мохнатых } \\
\text { '(language) } \\
\text { of the hairy } \\
\text { people' } \\
\text { in Cyrillic } \\
\text { = Southern } \\
\text { Kuril Ainu }\end{array}$ & Interpretation \\
\hline 39. женщина & woman & мать & mat & миного & menoko \\
\hline 4о. мальчикъ & boy & пумбо & $\begin{array}{l}\text { pon-po } \\
\text { small-child }\end{array}$ & $\Gamma^{\mathrm{h}}$ егаджи & hekaci \\
\hline 41. дњвочка & girl & кама & kamas & оберги & operke \\
\hline 42. отецъ & father & & & миджи & mici \\
\hline 43. мать & mother & & & нонну & nonno ${ }^{\mathrm{h}}$ \\
\hline 44. мужъ & husband & & & окай & okay \\
\hline 45. жена & wife & & & хмаджи & $\begin{array}{l}\text { ku-mac-i } \\
\{1 \mathrm{SG} . \\
\text { Poss- } \\
\text { woman- } \\
\text { AFF }\}\end{array}$ \\
\hline 46. братъ & brother & & & обу & hapo $^{\mathrm{i}}$ \\
\hline 47. сестра & sister & & & мачеръ ба & macirpe \\
\hline 48. сынъ & son & смотри маль- & & & \\
\hline 49. дочь & daughter & $\begin{array}{l}\text { чикъ и дьвочка } \\
\text { 'see boy and girl' }\end{array}$ & & & \\
\hline 5о. голова & head & & & па & $p a$ \\
\hline 51. рука & hand & & & текъ & tek \\
\hline 52. глаза & eyes & & & сикъ & sik \\
\hline 53. носъ & nose & & & эду & etu \\
\hline 54. губы & lips & чадай & catoy & чибусъ & capus \\
\hline
\end{tabular}

g This could be a borrowing from Japanese kama 'wife' (Nihon daijiten kankōkai 1973). Cf. many Ainu dialects use the Japanese word 39. menoko 'woman' so using a Japanese word for 'girl' would not be unusual.

$\mathrm{h}$ In Kuril Ainu, nonno is 'mother' (Dybowski 1892: 46, Torii 19o3:66). Cf. Hokkaido Ainu nonno 'flower, something nice (e.g. a toy) in children's language' (Tamura 1996: 433).

i See hapo in Torii (1903: 67); cf. in Hokkaido Ainu, hapo means 'mother' or 'father' depending on a particular dialect (Hattori 1964: 39, 321). 
TABLE 1 Словарь Курильского языка [“A Kuril Ainu Glossary”] (cont.)

\begin{tabular}{|c|c|c|c|c|c|}
\hline $\begin{array}{l}\text { (Russian } \\
\text { gloss) }\end{array}$ & $\begin{array}{l}\text { English } \\
\text { translation }\end{array}$ & $\begin{array}{l}\text { русских } \\
\text { курильцев } \\
\text { '(language) of } \\
\text { the Russian } \\
\text { Kurils' in } \\
\text { Cyrillic } \\
\text { = Northern } \\
\text { Kuril Ainu }\end{array}$ & Interpretation & $\begin{array}{l}\text { мохнатых } \\
\text { '(language) } \\
\text { of the hairy } \\
\text { people' } \\
\text { in Cyrillic } \\
\text { = Southern } \\
\text { Kuril Ainu }\end{array}$ & Interpretation \\
\hline 55. ротъ & mouth & & & чаръ & car \\
\hline 56. нога & leg, foot & кема & kema & $\mathrm{t}^{\mathrm{th}}$ чингю & cinkew \\
\hline 57. языкъ & tongue & & & ay & $a w$ \\
\hline $\begin{array}{l}\text { 58. борода } \\
\text { (волосы) }\end{array}$ & $\begin{array}{l}\text { beard } \\
\text { (hair) }\end{array}$ & дрекъј & rek & ноткиръ & notkir ${ }^{\mathrm{k}}$ \\
\hline 59. зубы & teeth & & & имаки & $\begin{array}{l}\text { imak-i } \\
\{\text { tooth-AFF }\}\end{array}$ \\
\hline 6о.ноготь & nail & & & амъ & am \\
\hline 61. палецъ & finger & & & ассигбитъ & askepet \\
\hline 62. x-й & $\begin{array}{l}\text { male genitals } \\
\text { (obscenity) }\end{array}$ & & & ти & $c i$ \\
\hline 63. м-де & $\begin{array}{l}\text { loins, testicles } \\
\text { (obscenity) }\end{array}$ & & & нокь & $\begin{array}{l}\text { nok-i } \\
\{\text { testicles-AFF }\}\end{array}$ \\
\hline 64. п-а & $\begin{array}{l}\text { female } \\
\text { genitals } \\
\text { (obscenity) }\end{array}$ & & & $\mathrm{T}^{\text {th }}$ ИТъ & cit \\
\hline 65. ж-а & $\begin{array}{l}\text { buttocks } \\
\text { (obscenity) }\end{array}$ & & & осаракамъ & $\begin{array}{l}\text { osor-kam } \\
\text { \{buttocks- } \\
\text { flesh\} }\end{array}$ \\
\hline $\begin{array}{l}\text { 66. волосы } \\
\text { на голове }\end{array}$ & head hair & & & py & $r u^{1}$ \\
\hline 67. слюна & saliva & & & нонъ & non \\
\hline
\end{tabular}

j Cf. "trek" in other sources. Elaborating on Vovin (1993), who reconstructions *tr-clusters, Alonso de la Fuente (2021: $36-37$, item 31 ) speculates that this spelling in reality could reflect the alveolar trill (i.e., $/ \mathrm{r} /$ as in Spanish rosa or perro) in opposition to the tap (i.e., / / /). See more items like that below, e.g. 168/178/210 требичи (reppis) 'three', 207. трепа (repa) 'three year', and 225. дребунгары (rep-un-kur \{sea-attach.to-man\}) 'Northern Kuril Ainu'. Yet we should also consider another possibility: these notations may represent a kind of retroflex stop in initial positions (Hattori 1964: 34).

k notkir 'chin' (Hattori 1964: 7-8).

l Cf. Hokkaido Ainu otop $(i)$ (Hattori 1964: 2). 
TABLE 1 Словарь Курильского языка [“A Kuril Ainu Glossary”] (cont.)

\begin{tabular}{|c|c|c|c|c|c|}
\hline $\begin{array}{l}\text { (Russian } \\
\text { gloss) }\end{array}$ & $\begin{array}{l}\text { English } \\
\text { translation }\end{array}$ & $\begin{array}{l}\text { русских } \\
\text { курильцев } \\
\text { '(language) of } \\
\text { the Russian } \\
\text { Kurils' in } \\
\text { Cyrillic } \\
\text { = Northern } \\
\text { Kuril Ainu }\end{array}$ & Interpretation & $\begin{array}{l}\text { мохнатых } \\
\text { '(language) } \\
\text { of the hairy } \\
\text { people' } \\
\text { in Cyrillic } \\
\text { = Southern } \\
\text { Kuril Ainu }\end{array}$ & Interpretation \\
\hline 68. лисица & fox & тчачо & cawcaw? $?^{\mathrm{m}}$ & чиренокъ & cironnup \\
\hline $\begin{array}{l}\text { 69. бобръ } \\
\text { морской }\end{array}$ & beaver & & & лахъко & rakko \\
\hline 7о. сивучь & sea lion & & & этаспе & etaspe \\
\hline 71. нерпъ & seal & ткв ${ }^{\mathbf{w}} а р ь$ & tukar & тукаръ & tukar \\
\hline 72. собака & $\operatorname{dog}$ & & & ста & sita \\
\hline 73. волкъ & wolf & & & оргеу & horkew \\
\hline 74. китъ & & & & $\mathrm{T}^{\text {th }}$ рига & rika \\
\hline 75. косатка & killer whale & $\mathrm{T}^{\mathrm{th}}$ чипъ & сер & $\mathrm{T}^{\text {th }}$ чача & caca?n \\
\hline 76. медвьдь & bear & & & кимумгамы & $\begin{array}{l}\text { kim-un- } \\
\text { kamuy }^{\circ} \\
\text { \{mountains- } \\
\text { live.at-god }\end{array}$ \\
\hline $\begin{array}{r}\text { 77. лисицы } \\
\text { красная }\end{array}$ & red foxes & & & хюри & $\begin{array}{l}\text { hure } \\
\text { red }\end{array}$ \\
\hline $\begin{array}{l}\text { 78. чернобу- } \\
\text { рая }\end{array}$ & silver (foxes) & & & штумбе & situmpe $\mathrm{p}$ \\
\hline $\begin{array}{l}\text { 79. сиводу- } \\
\text { шка }\end{array}$ & $\begin{array}{l}\text { hybrid } \\
\text { between the } \\
\text { silver and the } \\
\text { red (foxes) }\end{array}$ & & & уткасури & etakasure?q \\
\hline
\end{tabular}

m In Hokkaido Ainu, cawcaw 'fox (Vulpes vulpes)' (Chiri 1976: 145), cf. in Kuril Ainu, chauchan 'fox' Torii (1903: 71), cf. čaača 'fox' (Dybowski 1892: 23), cacoo 'fox' (Voznesensky 1844: f. 53, op. 1, \#43, word no. 510).

n In Hokkaido Ainu, caca 'uncle', kamuy caca 'bear' (Hattori 1964: 36, 185); cf. čačani 'marine animal similar to a dolphine' (Dybowski 1892: 23).

o A loanword from Old Japanese kamï 'god', see $\$ 4.2$.

p The word situmpe (< situ-un-pe \{mountain.range-attach.to-thing\} 'fox (Vulpes vulpes schrencki Kishida)') is a polite name for a fox or the most highly ranked type of fox (Chiri 1976: 145).

q e-ta-kasu-re \{APPL-?-exceed-CAUS\} 'surpassing, special, unusual, extraordinary' (adverb) (Tamura 1996: 130), the same in Kayano (1996: 140), Kubodera (1992: 69), and Batchelor (1938: 135). This perfectly reflects the unusual color of this type of fox. 
TABLE 1 Словарь Курильского языка [“A Kuril Ainu Glossary”] (cont.)

\begin{tabular}{|c|c|c|c|c|c|}
\hline $\begin{array}{l}\text { (Russian } \\
\text { gloss) }\end{array}$ & $\begin{array}{l}\text { English } \\
\text { translation }\end{array}$ & $\begin{array}{l}\text { русских } \\
\text { курильцев } \\
\text { '(language) of } \\
\text { the Russian } \\
\text { Kurils' in } \\
\text { Cyrillic } \\
\text { = Northern } \\
\text { Kuril Ainu }\end{array}$ & Interpretation & $\begin{array}{l}\text { мохнатых } \\
\text { '(language) } \\
\text { of the hairy } \\
\text { people' } \\
\text { in Cyrillic } \\
=\text { Southern } \\
\text { Kuril Ainu }\end{array}$ & Interpretation \\
\hline 8о.рыба & fish & & & $\mathrm{T}^{\text {th }}$ чепъ & сер \\
\hline 81. рямша & sculpins & сокъ & $s o k^{r}$ & ч⿳⺈еби & $\begin{array}{l}\text { cep-i } \\
\{\text { fish-AFF }\}\end{array}$ \\
\hline 82. хайко & $\begin{array}{l}\text { Siberian } \\
\text { salmon, dog } \\
\text { salmon }\end{array}$ & & & сиби & $\begin{array}{l}\text { si-pe } \\
\text { \{true-thing\} }\end{array}$ \\
\hline 83. горбуша & $\begin{array}{l}\text { humpback } \\
\text { salmon }\end{array}$ & & & сагиби & $\begin{array}{l}\text { sak-ipe } \\
\text { \{summer- } \\
\text { food\} }\end{array}$ \\
\hline 84. нерка & red salmon & & & сижипъ & $\begin{array}{l}\text { si-cep } \\
\{\text { true-fish }\}\end{array}$ \\
\hline 85. свинка & dolphin & & & одумъ & okom?s \\
\hline 86. птица & bird & тчиръ & cir & тчипапъ & cikap \\
\hline 87. орелъ & eagle & сунгуръ & surkurt & кабачиръ & kapatcir \\
\hline $\begin{array}{c}\text { 88. альбат } \\
\text { росъ }\end{array}$ & albatross & & & оннидори & $\begin{array}{l}\text { onne-tori }{ }^{\mathrm{u}} \\
\{\text { big-bird }\}\end{array}$ \\
\hline 89. топорокъ & puffin & & & этубрига & etupirka \\
\hline 9о.штаны & pants & & & oio & $o y o^{\mathrm{v}}$ \\
\hline 91. рубашка & shirt & $\begin{array}{l}\text { ньтъ слова } \\
\text { 'no word' }\end{array}$ & & & \\
\hline 92. сапоги & boots & & & керъ & ker \\
\hline 93. тарбаса & $\begin{array}{l}\text { boots (used } \\
\text { on the Far } \\
\text { East) }\end{array}$ & & & керъ & ker \\
\hline 94. парка & coat & имми & imi & уръ & $u r$ \\
\hline
\end{tabular}

\footnotetext{
r sok 'fish, blob' Cottus (Dybowski 1892: 6o).

s okom 'dolphin' (including several species) (Chiri 1976: 174).

t surkur 'eagle' (Krasheninnikov 1755.2: 188, Torii 1903: 72).

$\mathrm{u}$ Borrowed from Japanese.

v oyo (Krasheninnikov 1755.2: 186), ojo, ojoo (Dybowski 1892: 48).
} 
TABLE 1 Словарь Курильского языка [“A Kuril Ainu Glossary”] (cont.)

\begin{tabular}{|c|c|c|c|c|c|}
\hline $\begin{array}{l}\text { (Russian } \\
\text { gloss) }\end{array}$ & $\begin{array}{l}\text { English } \\
\text { translation }\end{array}$ & $\begin{array}{l}\text { русских } \\
\text { курильцев } \\
\text { '(language) of } \\
\text { the Russian } \\
\text { Kurils' in } \\
\text { Cyrillic } \\
\text { = Northern } \\
\text { Kuril Ainu }\end{array}$ & Interpretation & $\begin{array}{l}\text { мохнатьх } \\
\text { '(language) } \\
\text { of the hairy } \\
\text { people' } \\
\text { in Cyrillic } \\
\text { = Southern } \\
\text { Kuril Ainu }\end{array}$ & Interpretation \\
\hline $\begin{array}{ll}\text { 95. } & \text { пшено } \\
& \text { проч. }\end{array}$ & millet etc. & & & амамъ & amam \\
\hline 96. дрова & & & & ни & $n i$ \\
\hline 97. черемша & & букса & pukusa & пукуса & pukusa \\
\hline 98. сарана & $\begin{array}{l}\text { Fritillaria } \\
\text { cam- } \\
\text { schatcensis: } \\
\text { Kamchatka } \\
\text { lily or wild } \\
\text { rice }\end{array}$ & сарана & sarana $^{\mathrm{w}}$ & apy & $a r u^{\mathrm{x}}$ \\
\hline 99. вино & wine & водка & votka ${ }^{\mathrm{y}}$ & саги & $s a k e^{\mathrm{z}}$ \\
\hline 10о. байдара & boat & тчипъ & $\operatorname{cip}$ & & \\
\hline $\begin{array}{l}\text { 101. бумага } \\
\text { писчая }\end{array}$ & $\begin{array}{l}\text { writing } \\
\text { paper }\end{array}$ & камбе & $k a n p i^{\mathrm{aa}}$ & & \\
\hline 102. табакъ & tobacco & тамбагу & tampaku ${ }^{\mathrm{ab}}$ & & \\
\hline 103. кур. трубка & $\begin{array}{l}\text { tobacco- } \\
\text { pipe }\end{array}$ & кес/шири & kiseri $^{\text {ac }}$ & киссири & kiseri \\
\hline 104. жельзо & iron & & & кани & kani \\
\hline 105. компасъ & compass & компасъ & kompas $^{\text {ad }}$ & & \\
\hline 106. ложка & spoon & & & пасв ${ }^{\mathrm{w}} и$ & pasuy $^{\mathrm{ae}}$ \\
\hline 107. ножъ & knife & & & эбиро & epira $^{\text {af }}$ \\
\hline
\end{tabular}

w Borrowed from Russian.

x Cf. arru 'a kind of lily, sarana, lilium tenuigolium' (Dybowski 1892: 19), haru 'Cardiocrinum Glehni Makino, Fritillaria kamtschatcensis Ker-Gawl' (Chiri 1976: 196, 223).

y Borrowed from Russian.

z $\quad$ Borrowed from Japanese.

aa Borrowed from Japanese.

$\mathrm{ab} \quad$ Borrowed from Japanese and into Japanese from Portuguese tabaco 'tobacco'.

ac Borrowed from Japanese and into Japanese from Portuguese que sorber 'smoke (a pipe)'.

ad Borrowed from Russian and into Russian from Italian compassio from compassare 'measure by steps'.

ae A loanword from Old Japanese pasï 'chopsticks', see §4.2.

af Also appears in Krasheninnikov (1755.2: 186) and Klaproth (1823: 309). 
TABLE 1 Словарь Курильского языка [“A Kuril Ainu Glossary”] (cont.)

\begin{tabular}{|c|c|c|c|c|c|}
\hline $\begin{array}{l}\text { (Russian } \\
\text { gloss) }\end{array}$ & $\begin{array}{l}\text { English } \\
\text { translation }\end{array}$ & $\begin{array}{l}\text { русских } \\
\text { курильцев } \\
\text { '(language) of } \\
\text { the Russian } \\
\text { Kurils' in } \\
\text { Cyrillic } \\
\text { = Northern } \\
\text { Kuril Ainu }\end{array}$ & Interpretation & $\begin{array}{l}\text { мохнатых } \\
\text { '(language) } \\
\text { of the hairy } \\
\text { people' } \\
\text { in Cyrillic } \\
\text { = Southern } \\
\text { Kuril Ainu }\end{array}$ & Interpretation \\
\hline 108. парусъ & sail & & & кая & kaya \\
\hline 109. порохъ & $\begin{array}{l}\text { gun- } \\
\text { powder }\end{array}$ & порохъ & poroh $^{\mathrm{ag}}$ & $\begin{array}{l}\text { уив }{ }^{\mathrm{w}} \text { и имо, } \\
\text { текопоку- } \\
\text { шири }\end{array}$ & $\begin{array}{l}\text { uipe } \text { imo }^{\text {ah }} \\
\text { \{pieces- } \\
\text { potato\} } \\
\text { teppo } \\
\text { kusuriai }^{\text {ai }} \\
\text { ggun- } \\
\text { medicine\} }\end{array}$ \\
\hline 110. ружье & gun & камойду & $\begin{array}{l}k a m u y^{\text {aj }} k u \\
\{\text { god bow }\end{array}$ & текъ-по & teppo \\
\hline 111. столь & table & парида & $\begin{array}{l}\text { para-ita }^{\text {ak }} \\
\text { \{wide- } \\
\text { board }\}\end{array}$ & & \\
\hline $\begin{array}{l}\text { 112. судно } \\
\text { мореходное }\end{array}$ & ship & легундо & rokunto ${ }^{\mathrm{al}}$ & пинза & pencay \\
\hline 113. топоръ & axe & & & мугаръ & mukar \\
\hline 114. свинецъ & lead & свинецъ & swinets $^{\mathrm{am}}$ & айнеканъ & aynep \\
\hline 115. чернилы & ink & камбенуитъ & $\begin{array}{l}\text { kanpi-nuye-p } \\
\text { \{paper-tattoo/ } \\
\text { write-thing\} }\end{array}$ & суми & sumi \\
\hline 116. юрта & house & & & $\mathrm{T}^{\mathrm{th}} \mathbf{\mathrm { Ye }}$ & $c e$ \\
\hline
\end{tabular}

\footnotetext{
ag Borrowed from Russian.

ah Borrowed from Japanese.

ai Both words are borrowed from Japanese.

aj A loanword from Old Japanese kamï 'god', see §4.2.

ak Borrowed from Japanese.

al A rare word found in yukar-epics in Hokkaido Ainu.

am Borrowed from Russian (Torii 1903: 71).
} 
TABLE 1 Словарь Курильского языка [“A Kuril Ainu Glossary”] (cont.)

\begin{tabular}{|c|c|c|c|c|c|}
\hline $\begin{array}{l}\text { (Russian } \\
\text { gloss) }\end{array}$ & $\begin{array}{l}\text { English } \\
\text { translation }\end{array}$ & $\begin{array}{l}\text { русских } \\
\text { курильцев } \\
\text { '(language) of } \\
\text { the Russian } \\
\text { Kurils' in } \\
\text { Cyrillic } \\
\text { = Northern } \\
\text { Kuril Ainu }\end{array}$ & Interpretation & $\begin{array}{l}\text { мохнатьх } \\
\text { '(language) } \\
\text { of the hairy } \\
\text { people' } \\
\text { in Cyrillic } \\
\text { = Southern } \\
\text { Kuril Ainu }\end{array}$ & Interpretation \\
\hline 117. пл? ботъ & ship, boat & & & $\begin{array}{l}\text { юрусъ } \\
\mathrm{T}^{\text {th }} \text { чипъ }\end{array}$ & $\begin{array}{l}\text { yar-us }{ }^{\text {an }} \text { cip } \\
\text { \{tree.bark- } \\
\text { attach boat }\}\end{array}$ \\
\hline 118. платокъ & shawl & & & кончи & konci \\
\hline 119. сабля & sable & & & эдообни & $\begin{array}{l}\text { etu-op-ni } \\
\{\text { beak-spear- } \\
\text { stick\} }\end{array}$ \\
\hline 12о. бълой & white & & & $\mathrm{T}^{\mathrm{th}}$ реторъ & retar \\
\hline 121. черной & black & & & эгурохъпе & $\begin{array}{l}\text { ekurok-pe } \\
\text { \{black-thing\} }\end{array}$ \\
\hline 122. красной & red & & & $\Gamma^{\mathrm{h}}$ уритке & $\begin{array}{l}\text { hure pe? } \\
\text { \{red thing }\end{array}$ \\
\hline $\begin{array}{l}\text { 123. болњзнь, } \\
\text { недугъ }\end{array}$ & illness & арга & arka & игони & ikoni \\
\hline 124. Я & I & & & кани & kani \\
\hline 125. ты & you & ани & eani & аногай & anokay \\
\hline 126. онъ & he & тангуръ & $\begin{array}{l}\tan k u r \\
\{\text { this person }\}\end{array}$ & туайнану & $\begin{array}{l}\text { taan/toan } \\
\text { aynu } \\
\text { \{this/ } \\
\text { that human\} }\end{array}$ \\
\hline 127. мы & we & & & айнодаръ & $\begin{array}{l}\text { aynu-utar } \\
\{\text { human-PL }\}\end{array}$ \\
\hline 128. вы & you (PL) & & & туанодаръ & $\begin{array}{l}\text { toan utar } \\
\text { \{this/ } \\
\text { that people }\}\end{array}$ \\
\hline
\end{tabular}

an Cf.yar-cip \{tree.bark-boat\} 'a boat made from tree bark' in Hokkaido Ainu (Kayano 1995: 45o, Kubodera 1992: 315). 
TABLE 1 Словарь Курильского языка [“A Kuril Ainu Glossary”] (cont.)

\begin{tabular}{|c|c|c|c|c|c|}
\hline $\begin{array}{l}\text { (Russian } \\
\text { gloss) }\end{array}$ & $\begin{array}{l}\text { English } \\
\text { translation }\end{array}$ & $\begin{array}{l}\text { русских } \\
\text { курильцев } \\
\text { '(language) of } \\
\text { the Russian } \\
\text { Kurils' in } \\
\text { Cyrillic } \\
\text { = Northern } \\
\text { Kuril Ainu }\end{array}$ & Interpretation & $\begin{array}{l}\text { мохнатых } \\
\text { '(language) } \\
\text { of the hairy } \\
\text { people' } \\
\text { in Cyrillic } \\
\text { = Southern } \\
\text { Kuril Ainu }\end{array}$ & Interpretation \\
\hline 129. они, онЊ & they & & & айнюгиръ & $\begin{array}{l}\text { aynu-ikir }{ }^{\text {ao }} \\
\{\text { human-PL }\}\end{array}$ \\
\hline 13०. Бшь & Eat! & кониби & $\begin{array}{l}\text { kon ipe } \\
\{\text { IMP eat }\}\end{array}$ & конибея & $\begin{array}{l}\text { kon ipe ya } \\
\{\text { IMP eat } \\
\text { IMP.POL }\}\end{array}$ \\
\hline 131. пей & Drink! & & & кумпегу & $\begin{array}{l}\text { kon pe-ku } \\
\{\text { IMP water- } \\
\text { drink }\}\end{array}$ \\
\hline $\begin{array}{r}\text { 132. хочу } \\
\text { пить }\end{array}$ & $\begin{array}{l}\text { I want to } \\
\text { drink }\end{array}$ & & & пехрейге & $\begin{array}{l}\text { pe-ku rusuy } \\
\{\text { water-drink } \\
\text { DESID }\}\end{array}$ \\
\hline 133. говорить & speak & & & кониданъ & $\begin{array}{l}\text { kon itak } \\
\{\text { IMP-speak }\}\end{array}$ \\
\hline 134. $\mathrm{e}-\mathrm{b}$ & $\begin{array}{l}\text { have a sexual } \\
\text { intercourse } \\
\text { (obscenity) }\end{array}$ & & & учавнасту & $\begin{array}{l}u \text {-ciw? } \\
\{\text { RECIP- } \\
\text { prick }\}\end{array}$ \\
\hline $\begin{array}{l}\text { 135. живъ, } \\
\text { здравст- } \\
\text { вуетъ }\end{array}$ & $\begin{array}{l}\text { be alive, } \\
\text { be healthy }\end{array}$ & & & вакнуа & $\begin{array}{l}\text { iwanke-an wa } \\
\left\{\text { healthy- } 4 \cdot^{\text {ap }}\right. \\
\text { S FIN }\}\end{array}$ \\
\hline 136. нездоровъ & be unhealthy & & & хейну & $\begin{array}{l}\text { hein } \\
\mathrm{NEG}^{\mathrm{aq}}\end{array}$ \\
\hline $\begin{array}{l}\text { 137. внутренняя } \\
\text { бользнь }\end{array}$ & $\begin{array}{l}\text { internal } \\
\text { illness }\end{array}$ & $\mathrm{B}^{\mathrm{w}}$ инуа & $\begin{array}{l}\text { wen wa } \\
\{\text { bad FIN }\}\end{array}$ & кигониуа & $\begin{array}{l}\text { ku-i-koniwa } \\
\{1 \mathrm{SG} . \mathrm{S}- \\
\text { ANTIP-suffer } \\
\text { FIN }\}\end{array}$ \\
\hline
\end{tabular}

138. плакать сry тисъ cis

ao ikir is a nominal plurality marker in Kuril Ainu.

ap 4th person is a common label for 1PL.INCL, 2SG/PL.HON, 1st person in oral literature or logophoric.

aq For the meaning 'unhealthy' the speaker probably just said 'no'. For a discussion of the negation marker in Kuril Ainu see Sato and Bugaeva (2019: 81). 
TABLE 1 Словарь Курильского языка [“A Kuril Ainu Glossary”] (cont.)

\begin{tabular}{|c|c|c|c|c|c|}
\hline $\begin{array}{l}\text { (Russian } \\
\text { gloss) }\end{array}$ & $\begin{array}{l}\text { English } \\
\text { translation }\end{array}$ & $\begin{array}{l}\text { русских } \\
\text { курильцев } \\
\text { ‘(language) of } \\
\text { the Russian } \\
\text { Kurils' in } \\
\text { Cyrillic } \\
\text { = Northern } \\
\text { Kuril Ainu }\end{array}$ & Interpretation & $\begin{array}{l}\text { мохнатых } \\
\text { '(language) } \\
\text { of the hairy } \\
\text { people' } \\
\text { in Cyrillic } \\
=\text { Southern } \\
\text { Kuril Ainu }\end{array}$ & Interpretation \\
\hline
\end{tabular}

\begin{tabular}{|c|c|c|c|c|c|c|}
\hline 139. & ты плачешь & you cry & этиса & $\begin{array}{l}e \text {-cis wa } \\
\{2 \text { SG.S-cry FIN }\}\end{array}$ & & \\
\hline 140. & пьть & sing & югаръ & yukar & & \\
\hline 141. & плясать & dance & дримси & rimse & & \\
\hline 142. & $\begin{array}{l}\text { попля- } \\
\text { ши }\end{array}$ & Dance! & кунъ дримси & kun rimse & & \\
\hline 143. & спать & sleep & & & могору & mokor \\
\hline 144. & садись & Sit down! & & & конабуна & $\begin{array}{l}\text { kon ahun ya } \\
\{\text { IMP enter. } \\
\text { SG IMP.POL }\}\end{array}$ \\
\hline 145. & стой & Stand! & & & конасъ & $\begin{array}{l}\text { kon as } \\
\{\text { IMP stand. } \\
\text { SG }\}\end{array}$ \\
\hline 146. & ты ударилъ & you hit & & & этюштагейки & $\begin{array}{l}\text { eci-u-sitayki } \\
(w) a \\
\{2 \text { PL.S- } \\
\text { RECIP- } \\
\text { hit FIN }\}\end{array}$ \\
\hline 147. & украсть & steal & & & иска & iska \\
\hline 148. & умереть & die & & & $\mathrm{T}^{\mathrm{th}}$ рай & ray \\
\hline 149. & смњяться & laugh & & & мина & mina \\
\hline $15 \circ$. & ты смъесся & you laugh & & & эмина & $\begin{array}{l}\text { e-mina } \\
\{2 \text { SG.s-laugh }\}\end{array}$ \\
\hline 151. & ссать & take a piss & & & осома & osoma \\
\hline $15^{2 .}$ & ср-ь & take a dump & & & коносома & $\begin{array}{l}\text { kon osoma } \\
\{\text { IMP defecate }\}\end{array}$ \\
\hline 153. & хорошо & good & & & пиргивª & $\begin{array}{l}\text { pirka wa } \\
\{\text { good FIN }\end{array}$ \\
\hline 154. & худо, дурно & bad & & & винв ${ }^{\mathrm{w}} \mathrm{a}$ & $\begin{array}{l}\text { wen wa } \\
\{\text { bad FIN }\}\end{array}$ \\
\hline
\end{tabular}


тAвLE 1 Словарь Курильского языка [“A Kuril Ainu Glossary”] (cont.)

\begin{tabular}{|c|c|c|c|c|c|}
\hline $\begin{array}{l}\text { (Russian } \\
\text { gloss) }\end{array}$ & $\begin{array}{l}\text { English } \\
\text { translation }\end{array}$ & $\begin{array}{l}\text { русских } \\
\text { курильцев } \\
\text { '(language) of } \\
\text { the Russian } \\
\text { Kurils' in } \\
\text { Cyrillic } \\
\text { = Northern } \\
\text { Kuril Ainu }\end{array}$ & Interpretation & $\begin{array}{l}\text { мохнатых } \\
\text { '(language) } \\
\text { of the hairy } \\
\text { people' } \\
\text { in Cyrillic } \\
\text { = Southern } \\
\text { Kuril Ainu }\end{array}$ & Interpretation \\
\hline 155. плевать & spit & & & нонету & $\begin{array}{l}\text { non-e-atu } \\
\text { \{saliva-with. } \\
\text { APPL-vomit }\end{array}$ \\
\hline 156. ты пьянъ & be drunk & & & $\begin{array}{l}\text { эсаги киндра- } \\
\text { гара }\end{array}$ & $\begin{array}{l}\text { e-sake-kinrakar } \\
\text { \{2SG.S-alcohol- } \\
\text { be.mad\} }\end{array}$ \\
\hline 157. Я пьянъ & I am drunk & & & $\begin{array}{l}\text { конисаги- } \\
\text { киндра- } \\
\text { гара }\end{array}$ & $\begin{array}{l}\text { kuani }(k u) \text {-sake- } \\
\text { kinrakar } \\
\text { \{1sG 1SG.S- } \\
\text { alcohol- } \\
\text { be.mad\} }\end{array}$ \\
\hline 158. сьверъ & North & $\begin{array}{l}\text { сисамъ- } \\
\text { чьянду }\end{array}$ & $\begin{array}{l}\text { sisam } \\
\text { si-anruru }^{\text {ar }} \\
\text { \{Japanese/ } \\
\text { foreigner } \\
\text { REFL-over.there }\end{array}$ & & \\
\hline $\begin{array}{l}\text { 159. сьверо- } \\
\text { востокъ }\end{array}$ & North East & мусиръ-ба & $\begin{array}{l}\text { mosir-pa } \\
\{\text { land-head }\}\end{array}$ & & \\
\hline 16о. востокъ & East & сирруръ & $\begin{array}{l}\text { sirur } \\
\text { ocean }\end{array}$ & & \\
\hline $\begin{array}{l}\text { 161. юго- } \\
\text { востокъ }\end{array}$ & South East & $\mathrm{B}^{\mathrm{w}}$ индрера & $\begin{array}{l}\text { wen rera }{ }^{\text {as }} \\
\{\text { bad wind }\}\end{array}$ & & \\
\hline 162. югъ & South & ояв ${ }^{\mathrm{w}}$ индрера & $\begin{array}{l}\text { oya wen rera } \\
\text { \{another bad } \\
\text { wind }\end{array}$ & & \\
\hline $\begin{array}{l}\text { 163. юго- } \\
\text { западъ }\end{array}$ & South West & мусиръ-гисъ & $\begin{array}{l}\text { mosir kes } \\
\{\text { land end }\end{array}$ & & \\
\hline 164. западъ & West & $\begin{array}{l}\text { маканургусъ- } \\
\text { оямъ }\end{array}$ & $\begin{array}{l}\text { makan-rur- } \\
\text { kes oya? } \\
\text { \{behind-ocean-end } \\
\text { another?\} }\end{array}$ & & \\
\hline
\end{tabular}

ar anruru 'over there' (Torii 1903: 74).

as Cf. v'ent'era 'eastern wind' (Dybowski 1892: 67). 
TABLE 1 Словарь Курильского языка [“A Kuril Ainu Glossary”] (cont.)

\begin{tabular}{|c|c|c|c|c|c|}
\hline $\begin{array}{l}\text { (Russian } \\
\text { gloss) }\end{array}$ & $\begin{array}{l}\text { English } \\
\text { translation }\end{array}$ & $\begin{array}{l}\text { русских } \\
\text { курильцев } \\
\text { '(language) of } \\
\text { the Russian } \\
\text { Kurils' in } \\
\text { Cyrillic } \\
\text { = Northern } \\
\text { Kuril Ainu }\end{array}$ & Interpretation & $\begin{array}{l}\text { мохнатых } \\
\text { '(language) } \\
\text { of the hairy } \\
\text { people' } \\
\text { in Cyrillic } \\
\text { = Southern } \\
\text { Kuril Ainu }\end{array}$ & Interpretation \\
\hline $\begin{array}{l}\text { 165. сьверо- } \\
\text { западъ }\end{array}$ & North West & макантуръ & $\begin{array}{l}\text { makan-rur at } \\
\{\text { behind-ocean }\}\end{array}$ & & \\
\hline 166. 1 & one & синипъ & $\begin{array}{l}\text { sine- } p \\
\{\text { one-thing.CL }\}\end{array}$ & & \\
\hline 167. 2 & two & тубичи & tuppis ${ }^{\mathrm{au}}$ & & \\
\hline 168. 3 & three & требичи & reppis ${ }^{\mathrm{av}}$ & & \\
\hline 169. 4 & four & инипъ & $\begin{array}{l}\text { ine- } p \\
\{\text { four-thing.CL }\}\end{array}$ & & \\
\hline 170. 5 & five & асигнипъ & $\begin{array}{l}\text { asikne-p } \\
\{\text { five-thing.CL }\}\end{array}$ & & \\
\hline 171. 6 & $\operatorname{six}$ & ив $^{\mathrm{w}} а \mathrm{aмъ}$ & iwan & & \\
\hline 172. 7 & seven & арувамби & $\begin{array}{l}\text { arwan-pe } \\
\{\text { seven-thing.CL }\}\end{array}$ & & \\
\hline 173. 8 & eight & тубисамбе & $\begin{array}{l}\text { tupesan-pe } \\
\text { \{eight-thing.CL }\end{array}$ & & \\
\hline 174. 9 & nine & синибисамбе & $\begin{array}{l}\text { sinepesan-pe } \\
\text { \{nine-thing\} }\end{array}$ & & \\
\hline 175. 10 & ten & вампе & $\begin{array}{l}\text { wan-pe } \\
\{\text { ten-thing.CL }\}\end{array}$ & & \\
\hline 176. 11 & eleven & $\begin{array}{l}\text { синипъ- } \\
\text { вампе- } \\
\text { казма }\end{array}$ & $\begin{array}{l}\text { sinep wan-pe } \\
\text { kasma }^{\text {aw }} \\
\text { \{one-thing.CL ten- } \\
\text { thing.CL leave }\end{array}$ & & \\
\hline
\end{tabular}

at Cf. makan ruru 'the northern sea' (Batchelor 1938: 289).

au Cf. tuppis 'two' for Yakumo (Hokkaido) in Hattori (1964: 26o).

av Cf. reppis 'three' for Yakumo (Hokkaido) in Hattori (1964: 260).

aw In most Hokkaido Ainu dialects (e.g. Saru and Chitose) the number 'eleven' (and some others) is expressed as sine-p i-kasma wan-pe \{one-thing.CL ANTIP-leave ten-thing.CL\} (Tamura 1996: 221, Nakagawa 1995: 29). 
TABLE 1 Словарь Курильского языка [“A Kuril Ainu Glossary”] (cont.)

\begin{tabular}{|c|c|c|c|c|c|}
\hline $\begin{array}{l}\text { (Russian } \\
\text { gloss) }\end{array}$ & $\begin{array}{l}\text { English } \\
\text { translation }\end{array}$ & $\begin{array}{l}\text { русских } \\
\text { курильцев } \\
\text { '(language) of } \\
\text { the Russian } \\
\text { Kurils' in } \\
\text { Cyrillic } \\
\text { = Northern } \\
\text { Kuril Ainu }\end{array}$ & Interpretation & $\begin{array}{l}\text { мохнатых } \\
\text { '(language) } \\
\text { of the hairy } \\
\text { people' } \\
\text { in Cyrillic } \\
\text { = Southern } \\
\text { Kuril Ainu }\end{array}$ & Interpretation \\
\hline 177. 12 & twelve & $\begin{array}{l}\text { тубичи- } \\
\text { вампе- } \\
\text { казма }\end{array}$ & $\begin{array}{l}\text { tuppis wan-pe } \\
\text { kasma } \\
\text { \{two ten-thing. } \\
\text { CL leave }\end{array}$ & & \\
\hline 178. 13 & thirteen & $\begin{array}{l}\text { требичи- } \\
\text { вампе- } \\
\text { казма }\end{array}$ & $\begin{array}{l}\text { reppis wan-pe } \\
\text { kasma } \\
\text { \{three ten-thing.PL } \\
\text { leave\} }\end{array}$ & & \\
\hline 179. 14 & fourteen & $\begin{array}{l}\text { инипъ- } \\
\text { вампе- } \\
\text { казма }\end{array}$ & $\begin{array}{l}\text { inep wan-pe kasma } \\
\text { \{four-thing.CL ten- } \\
\text { thing.CL leave }\end{array}$ & & \\
\hline 18o. 15 & fifteen & $\begin{array}{l}\text { асигнипъ- } \\
\text { вампе- } \\
\text { казма }\end{array}$ & $\begin{array}{l}\text { asikne-p wan-pe } \\
\text { kasma } \\
\text { \{five-thing.CL } \\
\text { ten-thing.CL leave }\end{array}$ & & \\
\hline 181. 16 & sixteen & $\begin{array}{l}\text { ивамъ }[\mathrm{w}]- \\
\text { вампе- } \\
\text { казма }\end{array}$ & $\begin{array}{l}\text { iwan wan- } \\
\text { pe kasma } \\
\text { \{six ten-thing.cL } \\
\text { leave }\end{array}$ & & \\
\hline 182. 17 & seventeen & $\begin{array}{l}\text { арувамбе- } \\
\text { вампе- } \\
\text { казма }\end{array}$ & $\begin{array}{l}\text { arwan-pe wan-pe } \\
\text { kasma } \\
\text { \{seven-thing.CL } \\
\text { ten-thing.CL leave\} }\end{array}$ & & \\
\hline 183. 18 & eighteen & $\begin{array}{l}\text { тубисамбе- } \\
\text { вампе- } \\
\text { казма }\end{array}$ & $\begin{array}{l}\text { tupesan-pe wan-pe } \\
\text { kasma } \\
\text { \{eight-thing.CL } \\
\text { ten-thing.CL leave\} }\end{array}$ & & \\
\hline 184. 19 & nineteen & $\begin{array}{l}\text { синибесамбе- } \\
\text { вампе- } \\
\text { казма }\end{array}$ & $\begin{array}{l}\text { sinepesan-pe wan- } \\
\text { pe kasma } \\
\text { \{nine-thing.cL ten- } \\
\text { thing.CL leave }\end{array}$ & & \\
\hline
\end{tabular}


TABLE 1 Словарь Курильского языка [“A Kuril Ainu Glossary”] (cont.)

\begin{tabular}{|c|c|c|c|c|c|}
\hline $\begin{array}{l}\text { (Russian } \\
\text { gloss) }\end{array}$ & $\begin{array}{l}\text { English } \\
\text { translation }\end{array}$ & $\begin{array}{l}\text { русских } \\
\text { курильцев } \\
\text { '(language) of } \\
\text { the Russian } \\
\text { Kurils' in } \\
\text { Cyrillic } \\
\text { = Northern } \\
\text { Kuril Ainu }\end{array}$ & Interpretation & $\begin{array}{l}\text { мохнатых } \\
\text { '(language) } \\
\text { of the hairy } \\
\text { people' } \\
\text { in Cyrillic } \\
\text { = Southern } \\
\text { Kuril Ainu }\end{array}$ & Interpretation \\
\hline 185. 20 & twenty & ходъ & hot & & \\
\hline 186. 21 & twenty one & $\begin{array}{l}\text { синипъ- } \\
\text { ходъ- } \\
\text { казма }\end{array}$ & $\begin{array}{l}\text { sine-p hot kasma } \\
\text { \{one-thing.CL } \\
\text { twenty leave }\end{array}$ & & \\
\hline 187. 22 & twenty two & $\begin{array}{l}\text { тубичи-ходъ- } \\
\text { казма } \\
\text { и проч. 'etc.' }\end{array}$ & $\begin{array}{l}\text { tuppis hot kasma } \\
\{\text { two twenty leave }\}\end{array}$ & & \\
\hline 188. 30 & thirteen & вамбедъ-ходъ & $\begin{array}{l}\text { wan-pe }(e)-t u \text {-hot } \\
\text { \{ten-thing.CL } \\
\text { (APPL)- } \\
\text { two-twenty\} }\end{array}$ & & \\
\hline 189. 31 & thirty one & $\begin{array}{l}\text { синипъ- } \\
\text { вамбедъ- } \\
\text { ходъ-казма } \\
\text { и проч. 'etc.' }\end{array}$ & $\begin{array}{l}\text { sine-p wan-pe } \\
\text { (e)-tu-hot } \\
\text { \{one-thing. } \\
\text { CL ten-thing.CL } \\
\text { (APPL)-two-hot }\end{array}$ & & \\
\hline 190. 40 & forty & туходъ & $\begin{array}{l}\text { tu-hot } \\
\{\text { two-twenty }\end{array}$ & & \\
\hline 191. 41 & forty one & $\begin{array}{l}\text { сипипъ- } \\
\text { туходъ } \\
\text { казма }\end{array}$ & $\begin{array}{l}\text { sine-p tu-hot } \\
\text { kasma } \\
\text { \{one-thing.CL } \\
\text { two-twenty leave }\end{array}$ & & \\
\hline 192. $5^{\circ}$ & fifty & & & & \\
\hline 193. $5^{1}$ & fifty one & & & & \\
\hline 194. 6 o & sixty & треходъ & $\begin{array}{l}\text { re hot }\{\text { three } \\
\text { twenty\} }\end{array}$ & & \\
\hline 195. 70 & seventy & & & & \\
\hline 196. 8 o & eighty & инъходъ & $\begin{array}{l}\text { ine hot }\{\text { four } \\
\text { twenty }\}\end{array}$ & & \\
\hline
\end{tabular}


TABLE 1 Словарь Курильского языка ["A Kuril Ainu Glossary”] (cont.)

\begin{tabular}{|c|c|c|c|c|c|}
\hline $\begin{array}{l}\text { (Russian } \\
\text { gloss) }\end{array}$ & $\begin{array}{l}\text { English } \\
\text { translation }\end{array}$ & $\begin{array}{l}\text { русских } \\
\text { курильцев } \\
\text { '(language) of } \\
\text { the Russian } \\
\text { Kurils' in } \\
\text { Cyrillic } \\
\text { = Northern } \\
\text { Kuril Ainu }\end{array}$ & Interpretation & $\begin{array}{l}\text { мохнатых } \\
\text { '(language) } \\
\text { of the hairy } \\
\text { people' } \\
\text { in Cyrillic } \\
\text { = Southern } \\
\text { Kuril Ainu }\end{array}$ & Interpretation \\
\hline 197. 90 & ninety & & & & \\
\hline 198. 100 & hundred & асигнипъ-ходъ & $\begin{array}{l}\text { asikne-p hot } \\
\text { \{five-thing.CL } \\
\text { twenty\} }\end{array}$ & & \\
\hline 199. 120 & $\begin{array}{l}\text { hundred and } \\
\text { twenty }\end{array}$ & иванходъ & $\begin{array}{l}\text { iwan hot }\{\text { six } \\
\text { twenty\} }\end{array}$ & & \\
\hline 200.200 & two hundred & ванходъ & $\begin{array}{l}\text { wan hot }\{\text { ten } \\
\text { twenty\} }\end{array}$ & & \\
\hline 201. 400 & four hundred & туванходъ & $\begin{array}{l}\text { tu wan hot \{two ten } \\
\text { twenty\} }\end{array}$ & & \\
\hline
\end{tabular}

TABLE 2 A narrative following "A Kuril Ainu Glossary"

Жители съверныхъ курильскихъ острововъ подвластныхъ Россіи, и южныя находящихся во владьниіе Россіи японцевъ, говорятъ однимъ и тьмъ же языкомъ съ небольшой разницей его произношенія; но очень мало такихъ вещей, которыя имъли бы разныя названія; всь оные означены въ словарь.

Выраженія Куриловъ ложись спать - конирамигасъ гокки.
Inhabitants of the Northern Kuril Islands governed by Russia, and the Southern Islands that are held by the Japanese speak the same language with slight differences in pronunciation; however, there are few things that would have different names; all of them are marked in the dictionary.

Kuril expressions

Go to bed! - koniramigas gokki. 202. kon ?a hotke

a It must be some kind of verb. 
Поди ко мнъ - конеге

Поди прочь - оехти яма.

Годъ называется мадапи, а со щетом другое названіе имъетъ, на примеръ:

\section{2 года - тупа \\ 3-трепа \\ 4 - инипа}

При семъ надобно заметить, что у нихъ щетъ просто идетъ такъ:

2 - тубичи

3 - требичи

4-инипь

Но съ существительнымъ посльдней слогъ отнимается, на прим:

2 собаки ту-ста

3 - тре-ста у

4- ассигни-ста

тихой день - сирбиръ-га

гладкое море, тишина - атвига убега

сильная погода, буря - T $^{\text {th }}$ рера-руй

волненіе - атвига-в ${ }^{\mathbf{w}}$ ину

утреняя звъзда (венера) - нисасаубъ

вечерняя звъзда(венера) - скояучекета
Come to me! - konege 203. kon ekya

Go away! - oehti jama. 204. eci-isam na \{2PL.S-not.exist FIN $\}$

The year is called madapi 205. mata-pa \{winter-year\}, but with numbers there are other names, for example:

2 years - tupa 2o6. tu-pa \{two-year\}

3 - repa 207. re-pa \{three-year\}

4- inepa 208. ine-pa \{four-year\}

At the same time, it is necessary to note, that numbers on their own go like this:

2 - tupichi 209. tuppis

3 - trebichi 210. reppis

4-inip 211. inep

But with a noun, the last syllable

disappeared, for example:

2 dogs tu sita 212. tu seta \{two dog\}

3 - tre-sta 213. re sita \{three dog\}

4-assigni-sta 214. asikne sita \{four-dog\}

quiet day - sirbir-ka 215. sir-pirka

\{appearance-good\}

smooth sea, calm - atwiga ubega

216. atuy-ka oupeka \{sea-surface straight\}

strong weather, storm $-\mathbf{t}^{\text {th }}$ rera-ruj

217. rera-ruy \{wind-violent

roughness - atwiga-wenu 218. atuy-ka wen \{sea-surface bad\}

morning star (Venus) - nisasaub

219. nisat-sawot \{morning-escape\}

evening star

ckojaucheketa 220. ci-ko-ya-uk keta \{1PL.A-from. APPL-net-take.SG star $\}^{\text {b }}$

b вечерняя звъзда - скояучекета [skojautfeketa]: In a Japanese-Ainu dictionary compiled in 1848 in Otaru (Hokkaido), there is a form chikoyaukinochiu (Satō 1995: 92). This form is clearly related to скояучекета 
TABLE 2 A narrative following "A Kuril Ainu Glossary" (cont.)

за что меня бранишь? - генга энь = = жагаръ?

Видно, что ты собака - ста иге = шренуа

Это курильская брань, одна только и есть у нихъ, другой и ньтъ никакой.

Японцы нашихъ курильцевъ называютъ: яуншишамъ,

а мохнатыхъ - яунгуръ.

А мохнатые нашихъ курильцевъ называютъ: дребунгары

Японцы рускихъ называютъ агайсшто; а мохнатые и курильцы рускихъ называют сизамъ.
What are you scolding me for? - genga

en = zhakar? 221. henka ${ }^{\mathrm{c}}$ en-cak-kar? ${ }^{\mathrm{d}}$

\{why 1SG.o-punishment-make\}

I see that you are a dog - sta ige $=$

shrenua 222. sita e-ki sirine wa $\{\mathrm{dog}$ 1SG.A-do VIS.EV COP FIN\}

These are Kuril swear words, the only ones, they do not have anything else.

The Japanese call our Kuril people:

jaunshisham 223. ya-un-sisam \{landattach.to-Japanese/foreigner\}, while

the hairy people - jaunkur 224. ya-un-kur \{land-attach.to-person\}.

And the hairy people call our Kurils:

drebungary 225. rep-un-kur \{sea-attach.to\}.

The Japanese call Russians agj-sshito 226. akai hito \{red-person\}, and the hairy people and Kurils call Russians sizam 227. sisam.

[skojau t feketa], though the final part nociw 'star' is replaced by keta 'star', which is the corresponding non-cognate form in the Kuril dialect. It is not so easy to reconstruct the correct form for this, but from another variant recorded in Kumajirō Uehara's Ezogoshū (Sasaki and Tanimoto 2019: 275), namely yashinochiu, we can assume that the name of this star has some relation to ya 'net', since yashi means a kind of fishing carried out with two boats and a net. If so, we can assume that скояучекета [skojau t feketa] is *ci-ko-ya-uk keta \{1PL.A-from.APPL-net-take.SG star\}, which means 'the star of which we deprive a net'. Yet a further study should be carried out to find out the reason why this star is called so in Ainu.

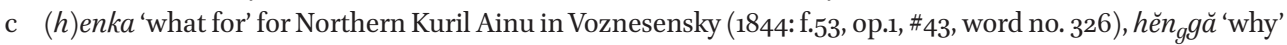
(Klaproth 1823: 52); cf. henkite 'why' (Torii 1903: 332).

d cak-kar \{punishment?-make\} is not registered in Ainu dictionaries but probably means 'to punish', cf. pak 'punishment' (Batchelor 1938: 377), which is related to a hotly disputed c:p phonological correspondence in Ainu dialects (Nakagawa and Fukazawa, to appear). 
TABLE 2 A narrative following “A Kuril Ainu Glossary” (cont.)

Что это такое? - г $\mathbf{h}$ амапъ_тамана?

Курильцы и махнатые не имњютъ названія лошади въ своемъ языкъ, а употребляютъ японское слово умма(мма) лошадь - а русскаго слова лошадь не знаютъ, но говорятъ конь.
What is this? - ghamap, tamana? 228. hemat, $^{\mathrm{e}}$ temana ${ }^{\mathrm{f}}$

The Kurils and hairy people do not have a word for "horse" in their language, and they use the Japanese word umma(mma) 229. umma лошадь, and they do not know the Russian word loshad', but they say kon' 23o. kon.

e hemat 'what' for Northern Kuril Ainu in Voznesensky (1844: f.53, op.1, \#43, word no. 1399, 1597, 1599).

f Cf. temana 'what' in Sakhalin Ainu (Hattori 1964: 314).

\section{$4 \quad$ Linguistic Characteristics of the Kuril Dialects Recorded in Golovnin (1811)}

According to Golovnin (1816: 258), "Ainu inhabitants of Hokkaido are different from Ainu inhabitants of Kuril Islands and have their own language. Though Hokkaido Ainu contains many Kuril Ainu words, it is incomprehensible to the Kuril Ainu. While Alexey and the inhabitants of Iturup and Kunashir could communicate freely, he could communicate with the Hokkaido Ainu with great difficulty, and sometimes even could not understand them. However, there is no doubt that all of them had been a single ethnos in the past: their appearance, customs and many similar words sufficiently testify about that." ${ }^{\prime 9}$ In the ensuing subsections, we are going to analyze in detail Golovnin's (1811) linguistic data and among other things to test them to the above-mentioned observations.

9 “Курильцы острова Мацмай отчасти особенный народ от жителей других Курильских островов и имеют свой язык, в котором хотя и есть много курильских слов, но курильцы их понимать не могут. Алексей и жители островов Итуруп и Кунасири совершенно разумели друг друга, но с мацмайскими курильцами он объяснялся с большим трудом, а иногда и понимать их не мог; но в том, что все они некогда составляли один и тот же народ, нет ни малейшего сомнения: наружный вид, обычаи и множество сходных слов в их языках слишком достаточно о том свидетельствуют." (Golovnin 1816: 258). 


\subsection{Lexical Features: Native Lexicon}

As seen from Table 3 below, there are clear lexical differences between the Northern and Southern Kuril dialects. Roughly speaking, the Southern Kuril dialects exhibit a greater lexical similarity to the Northeastern Hokkaido dialects. This makes us reconsider the above-mentioned Golovnin's claim and the claim by Murayama (1971: 77) that linguistically the Northern and Southern Kuril dialects are related more closely to each other than to the Hokkaido dialects. Furthermore, the similarity of the Southern Kuril dialects to the Hokkaido dialects can also be found in an old Japanese document called Etorofu omusha moshiwatashi [An Ainu translation of orders by the Japanese authorities on Etorofu (Iturup) Island] (1821) (held in the Archives of Hokkaido). This supports once again our observation that the Southern Kuril dialects had more lexical resemblance to the Hokkaido dialects, especially Eastern Hokkaido dialects (e.g. Bihoro in Table 3). On the other hand, we should also be aware that in some cases, forms in Golovnin's Southern Kuril dialect materials bear a striking resemblance to the Northern Kuril dialect collected by Torii in 1903, for example, нонну 'mother' and обу 'brother' in Table 3. Therefore, from this fact we may say that although the Southern Kuril dialects exhibits a strong similarity to the Northeastern Hokkaido dialects in a number of respects, they may nevertheless have undergone many particular changes common to the Northern Kuril dialects too.

TABLE 3 A comparison of Northern and Southern Kuril Ainu with Hokkaido Ainu

\begin{tabular}{|c|c|c|c|c|}
\hline & $\begin{array}{l}\text { Northern } \\
\text { Kuril } \\
\text { dialects } \\
\text { (Golovnin } \\
1811 \text { ) }\end{array}$ & $\begin{array}{l}\text { Northern } \\
\text { Kuril dialects } \\
\text { (Torii 1903) }\end{array}$ & $\begin{array}{l}\text { Southern } \\
\text { Kuril dialects } \\
\text { (Golovnin } \\
\text { 1811) }\end{array}$ & $\begin{array}{l}\text { Hokkaido dialects } \\
\text { (Hattori 1964) }\end{array}$ \\
\hline 87. eagle & $\begin{array}{l}\text { сунгуръ } \\
\text { (sungur) }\end{array}$ & shurukuru & $\begin{array}{l}\text { кабачиръ } \\
\text { (kabachir) }\end{array}$ & kapatcir (Bihoro dialect) \\
\hline 21. water & ппе (ppe) & peh & $\begin{array}{l}\text { вакка } \\
\text { (wakka) }\end{array}$ & wakka (Bihoro dialect) \\
\hline 39. woman & мать (mat) & mat & $\begin{array}{l}\text { миного } \\
\text { (minago) }\end{array}$ & menoko (Bihoro dialect) \\
\hline 43. mother & & nonno & $\begin{array}{l}\text { нонну } \\
\text { (nonnu) }\end{array}$ & hapo (Bihoro dialect) \\
\hline
\end{tabular}


TABLE 3 A comparison of Northern and Southern Kuril Ainu with Hokkaido Ainu (cont.)

\begin{tabular}{|c|c|c|c|c|}
\hline & $\begin{array}{l}\text { Northern } \\
\text { Kuril } \\
\text { dialects } \\
\text { (Golovnin } \\
1811 \text { ) }\end{array}$ & $\begin{array}{l}\text { Northern } \\
\text { Kuril dialects } \\
\text { (Torii 1903) }\end{array}$ & $\begin{array}{l}\text { Southern } \\
\text { Kuril dialects } \\
\text { (Golovnin } \\
\text { 1811) }\end{array}$ & $\begin{array}{l}\text { Hokkaido dialects } \\
\text { (Hattori 1964) }\end{array}$ \\
\hline 46. brother & & habo & $\begin{array}{l}\text { обу } \\
\text { (obu) }\end{array}$ & mici (Bihoro dialect) \\
\hline 54. lips & $\begin{array}{l}\text { чадай } \\
\text { (chadaj) }\end{array}$ & chātoi & $\begin{array}{l}\text { чибусъ } \\
\text { (chibus) }\end{array}$ & capus (Bihoro dialect) \\
\hline 68. fox & $\begin{array}{l}\text { тчачо } \\
\text { (tchacho) }\end{array}$ & chauchau & $\begin{array}{l}\text { чиренокъ } \\
\text { (chirenok) }\end{array}$ & $\begin{array}{l}\text { cironnup (Bihoro } \\
\text { dialect) }\end{array}$ \\
\hline 86. bird & tчиръ (tchir) & chiri & $\begin{array}{l}\text { тчипапъ } \\
\text { (tchipap) }\end{array}$ & cikap (Bihoro dialect) \\
\hline 40. boy & $\begin{array}{l}\text { пумбо } \\
\text { (pumbo) }\end{array}$ & bombo & $\begin{array}{l}\Gamma^{\mathrm{h}} \text { егаджи } \\
\text { (ghegadži) }\end{array}$ & $\begin{array}{l}\text { hekaci (Saru dialect), } \\
\text { 'ekaci (Bihoro dialect) }\end{array}$ \\
\hline
\end{tabular}

\subsection{Lexical Features: Borrowed Lexicon}

There are quite many borrowings in Kuril Ainu, especially in the category 100.-119. Tools and utensils (Table 1). It would be natural to expect that there are more borrowings from Russian in the Northern Kuril Ainu and less in Southern Kuril Ainu, while in Southern Kuril Ainu, the situation is opposite. However, this is not necessarily the case. The total number ${ }^{10}$ of borrowings from Russian in Kuril Ainu is 6, and indeed all of them were borrowed into Northern Kuril Ainu as in (1). The total number of borrowings from Japanese is 14: 5 into Northern Kuril Ainu and 9 into Southern Kuril Ainu (NK = Northern Kuril; SK = Southern Kuril) as in (2) and (3), which probably means that the Japanese and Northern Kuril Ainu never stopped trading.

(1) 98. сарана sarana 'Kamchatka lily' (NK)

99. водка votka 'wine' (NK)

105. компасъ kompas 'compass' (NK)

109. порохъ poroh 'gunpowder' (NK)

10 We count the number of words with borrowed items, not the number of borrowed items. When there are more than two morphemes/words in borrowings, the borrowed item is shown in bold. 
114. свинецъ swinets 'lead' (NK)

23о. конь kon' 'horse' (NK)

(2) 101. камбе kanpi 'writing paper' (NK)

102. тамбагу tampaku 'tobacco' (NK)

103. кес/шири kiseri 'tobacco-pipe' (NK)

111. парида para-ita \{wide-board\} 'table' (NK)

115. камбенуитъ kanpi-nuye-p \{paper-tattoo/write-thing\} 'ink' (NK)

(3) 99. саги sake 'wine' (SK)

103. киссири kiseri 'tobacco-pipe' (SK)

104. кани kani 'iron' (SK)

106. пасв ${ }^{\mathbf{w}}$ и pasuy 'spoon' (SK)

109. уив ${ }^{\text {} и ~ и м о ~ u i p e ~ i m o ~\{p i e c e s-p o t a t o\} ~ ' g u n p o w d e r ' ~(S K) ~}$

109.текопокушири teppo kusuri \{gun-medicine\} 'gunpowder' (SK)

110. текъ-по tерро 'gun'(SK)

115. суми sumi 'ink' (SK)

229. умма(мма) umma 'horse' (SK?)

There are also some very old borrowings from Japanese, for example kamuy 'god' (\#5, 76, 110) or pasuy 'chopsticks', here 'spoon' (\#106), which can be dated back to Old Japanese (70o-8oo), ${ }^{11}$ i.e. before the time the Ainu moved from Hokkaido to the Kurils.

\subsection{Phonetic Features}

1) In a few cases, the Kuril dialects show the devoicing or reduction of vowels between voiceless consonants, which is not prevalent in Hokkaido dialects:

11 The Japanese mï in kamï (神) is a so-called 'otsurui' group vowel of Old Japanese (Jōdaigo jiten henshū iinkai 1967: 219, 696). Therefore, the Ainu uy may be a result of this fact and if this is the case, this borrowing should be dated to a very ancient period. Moreover, as mentioned, there is another interesting example like this: pasuy 'chopsticks' 「箸」. This example shows a phonetic parallelism with kamuy and muy, but since「し」 (shi) is commonly believed to lack the distinction of the kó-otsu groups (here, $i$ vs. $i$ ) in kana, we cannot explain this peculiar form by referring to the kana-distinction. If there had been a similar kana-distinction in $\lceil し 」$ in very old times as well, the Ainu form could mirror this unknown historical fact. In fact, Mabuchi (1953) claims that there was such a distinction of two different 「し」 at least in $\operatorname{Kojiki}(712)$ : cf. the kō-group「斯, 紫, etc.」 (with $i$ ), the otsu-group「志, 芝, etc.」(with $i$ ). For example, hasi「箬」 is written as「波 志」(Jōdaigo jiten henshū iinkai 1967), where the second syllable is the otsu-group kana 「志」(i), which can explain why the Ainu pasuy 'chopsticks' (or 'spoon' here) has such a "strange" form now. 
(4) 71. ткварь (tkvw ar') (NK) 'seal' vs. tukar (Hokkaido dialect) (cf. тукаръ $(\mathrm{SK}))$

72. ста (SK) (sta) 'dog' vs. sita 'dog' (Hokkaido dialect) (Hattori 1964: 186) 78. штумбе (Jtumbe) (SK) 'silver (foxes)' vs. situmpe (Hokkaido dialect) (Chiri 1976: 145)

116. $\mathbf{T}^{\text {th }} \mathbf{\text { чe }}(\mathrm{SK})\left(\mathrm{t}^{\text {th }} \mathrm{t} \mathrm{e}\right.$ ) 'house' vs. cise 'house' (Hokkaido dialect) (Hattori 1964: 100)

2) A general accentuation rule in Ainu is as follows: if the first syllable is closed (heavy) then it is accented as in 21. wák.ka 'water', if the first syllable is open (light) then the accent falls on the second syllable as in 94. i.mi 'coat'. Among all Hokkaido dialects, the Kuril Ainu dialects are claimed to most closely resemble the Northeastern Hokkaido dialects, which are famous for the reduction of $/ \mathrm{h} /$ occurring before an unaccented vowel, e.g. the word he.káci 'boy' (Southwestern Hokkaido) would usually become $\boldsymbol{e} . k a ́ c i$ 'boy' in EH. However, the reduction of /h/ before an unaccented vowel seems to be uncommon in Kuril Ainu, which is proved by Golovnin's materials (5).

(5) 40. $\mathbf{r}^{\mathbf{h}}$ егаджи (g $\mathbf{g}^{\mathbf{h}}$ egaji) he.káci 'boy' (SK), see also ге́качичиръ (gekachichir) he.káci-cir \{boy-bird\} 'старики' (an unknown bird) (Krasheninnikov 1755.2: 188), cf. ekáci 'boy' in EH

In fact, there are words without the reduction of $/ \mathrm{h} /$ before an unaccented vowel in other Kuril Ainu materials too:

(6) а. гураи (gurai) hu.ráye 'wash' (Voznesensky 1844: f.53, op.1, \#43, word no. 87), but urai in Dybowski (1892: 66), which is an exception, cf. uráye 'wash' in EH;

b. kani haiyoku'pe kani ha.yókpe 'armour' \{metal-armour\} (Torii 19o3: 71), cf. ayókpe in $\mathrm{EH}$

Another even more striking feature of Kuril Ainu is that it quite unexpectedly shows the reduction of $/ \mathrm{h} /$ in accented (heavy) syllables both in Golovnin's (7a) and other materials $(7 \mathrm{~b}-\mathrm{c})$, which never occurs in $\mathrm{EH}$ or any other Ainu variety.

(7) a. 73. оргеy (orgeu) ór.kew 'wolf' (SK), see also opriy (orgiu) (Krasheninnikov 1755.2: 188), stōrkky̆gh (sta orkew) \{dog-wolf\} (Klaproth 1823: 314), orkiu (Dybowski 1892: 49), cf. hórkew 'wolf' in all Hokkaido and Sakhalin dialects 
b. арги (argi) ár.ki 'left' (Voznesensky 1844: f.53, op.1, \#43, word no. 534), arki (Dybowski 1892: 19), but hârky̆ in Klaproth (1823: 309), which is an exception, cf. harki 'left' in all Hokkaido and Sakhalin dialects

c. апрапъ (aprap) áp.rap 'March' (Voznesensky 1844: f.53, op.1, \#43, word no. 1551), aprap (Dybowski 1892: 19), cf. haprap 'February/March' as attested in some Hokkaido dialects

It is quite likely that historically this reducible /h/ in heavy syllables of Kuril can be traced back to a different consonant ${ }^{*} \mathrm{X}$ whose exact nature is unclear but we may try to speculate that it was some kind of glide.

Importantly, in Golovnin's data, the reduction of $/ \mathrm{h} /$ does not occur in monosyllabic words consisting of heavy syllables as in (8).

(8) а. 3о. $\mathbf{r}^{\mathbf{h}}$ уръ ( $\mathbf{g}^{\mathbf{h}}$ ur) húr 'mountain' (SK), cf. hur 'mountain' in some Hokkaido dialects

b. 5. камих ${ }^{\mathbf{h}}$ юмъ (kamih' hum 'thunder' in all Hokkaido and Sakhalin dialects

c. 185. ходъ (hod) hót 'twenty' (NK), cf. hot 'twenty' in all Hokkaido and Sakhalin dialects

3) non-occurrence of a parasitic vowel after a syllable final /r/. In a number of Hokkaido dialects, it is very common that a parasitic vowel arises after a syllable final /r/. In Sakhalin dialects, this parasitic vowel developed into an independent vowel phoneme functioning as a kind of formative of the word:

(9) kor [kor $\sim$ koro] (Hokkaido dialect), cf. koro (Sakhalin dialect) (Hattori 1964: 150)

Importantly, such parasitic vowels do not occur so often in our materials:

(10) 86. тчиръ (tchir) 'bird' (NK)

92. керъ (ker) 'boots' (SK)

123. арга (arga) 'illness' (NK)

94. уръ (ur) 'coat' (SK)

However, one example with a parasitic vowel in the word-final position was also attested in the Southern Kuril dialect:

(11) 156. эсаги киндра-гара (esagi kindra-gara) 'to be drunk' ("e-sake-kinra$\operatorname{kar}\{2 \mathrm{SG} . \mathrm{S}$-alcohol-be.mad $\})(\mathrm{SK})$ 
This example may show that the tendency was in the process of spreading (= change in progress) first in the Southern Kuril dialects, perhaps under the influence of the Hokkaido dialects.

Overall it is clear that although on the lexical level Kuril Ainu is close to Eastern Hokkaido Ainu (e.g. Bihoro dialect in Table 3), on the phonological level it is quite different from the latter.

\subsection{Grammatical Features}

Of course, it is not so easy to decide on the exact nature of grammar in these Kuril dialects only based on our insufficient data. However, we could find a few forms which are very important for considering dialectal peculiarities in grammar in the Kuril dialects:

1) Imperative is often marked by a special form ("kon *kun) that is not attested in other Ainu dialects:

(12) 13о. конибея (konosoma) 'Eat!' (“kon ipe ya) (SK)

13о. кониби (konibi) 'Eat!' (“kon ipe) (NK)

133. кониданъ (konidan) 'speak' (*kon itak) (SK)

144. конабуна (konabuna) 'Sit down/enter!' (“kon ahun ya) (SK)

145. конасъ (konas) 'Stand!' ( ${ }^{*}$ kon as) (SK)

152. коносома (konosoma) 'take a dump' ("kon osoma) (SK)

203. конеге (konege) 'Come to me!' ( ${ }^{*}$ kon ek ya) (NK?)

(13) 142. кунъ дримси (kun drimshi) 'Dance!' (“kun rimse) (NK) 131. кумпегу (kumpeku) ‘Drink water!' (*kun pe-ku) (SK)

We could not find this form attested anywhere else so far but for the time being it may suffice to say that it might be related to the form kunnano 'to be early; quickly' found in the Asahikawa dialect (Hattori 1964: 253), which also belongs to the Northeastern Hokkaido dialects. This form may be analyzed into *kun(root), -na (presumably a predicate-forming suffix, cf. Chiri 1974: 42, 119), -no (adverbial suffix). The root kun- may be historically associated with our reconstructed imperative marker *kon *kun.

Incidentally, it should be noted that in this case the sentence final imperative particle is ya (not yan as in most Hokkaido dialects), which is also attested in the Asahikawa dialect (Hattori 1964: 333).

2) Numeral expressions found in Golovnin's materials exhibit a different construction as compared with other Ainu dialects. In Torii (1903), we can find such a form as shine wambe kasuma \{one ten leave\} 'eleven'. But 
such a form has not ever been reported for any other Ainu dialect. Usually, 'eleven' is expressed by a form like sinep ikasma wanpe \{one remain ten\} in the other Ainu dialects, for details see note aw in Table 1. However, here 'eleven' is 176. синипъ-вампе-казма *sinep wanpe kasma ${ }^{12}$ \{one ten leave $(\mathrm{NK})$ just as in Torii (1903: 124). We cannot explain the reason for this dialectal difference. But Golovnin's materials suggest that we need to take up Torii's description again and consider in detail this peculiarity both from a synchronic and diachronic point of view.

In this paper, we have clearly shown that Southern Kuril has a much closer lexical resemblance to Northeastern Hokkaido Ainu than Northern Kuril, see Table (3). Also, Southern Kuril Ainu only occasionally shows the insertion of a parasitic vowel after a syllable final $/ \mathrm{r}$ / being the influence of Hokkaido Ainu, which has not extended to Northern Kuril.

On the other hand, this paper reaffirmed that both Southern and Northern Kuril Ainu constitute a really separate Kuril group because they show a number of lexical, phonological and grammatical features, which are different from Northeastern Hokkaido Ainu. For example, in lexicon, Kuril Ainu employs nonno for 'mother' and hapo for 'brother', while the former word means 'flower or something nice' in Hokkaido Ainu and the latter means 'father' or 'mother' depending on a particular Hokkaido dialect. In the phonology, Kuril Ainu, unlike Northeastern Hokkaido Ainu, exhibits such phenomena as the devoicing or reduction of vowels between voiceless consonants, a unique phenomenon of the reduction of $/ \mathrm{h} /$ in accented (heavy) syllables, but does not exhibit such common phenomena as the reduction of $/ \mathrm{h} /$ before an unaccented vowel and the insertion of a parasitic vowel after a syllable final $/ \mathrm{r} /$. In the grammar, Kuril Ainu employs a special imperative form, i.e. a pre-verbal *kon *kun, which has a cognate in the Asahikawa (Northeastern Hokkaido) dialect, and a very peculiar construction for encoding 'eleven' and the like numbers, which is not attested elsewhere. It is obvious that Southern Kuril has undergone many particular changes common to Northern Kuril dialect, which made both distant from Hokkaido Ainu.

We have also examined borrowings in Kuril Ainu from Russian and Japanese. It appears that there are almost three times more borrowings from

12 Kasma 'leave' and $i$-kasma \{ANTIP-leave\} 'remain' are a transitive-intransitive pair, the antipassive $i$ - blocks the expression of the object (Bugaeva 2021). 
Japanese (14 items) than from Russian (6 items), including even 5 items from Japanese in Northern Kuril Ainu, which is probably indicative of the trade scale of the respective countries of those days.

\section{Acknowledgements}

We would like to thank the reading room staff of the Russian National Archive of the Navy in St. Petersburg, especially E. V. Nikandrova and E. L. Bychkova, for their kind cooperation, and a PhD student Anastasiya Gorlova (University of Missouri) for helping us to type Cyrillic materials. We would like to express our deep gratitude to Olga Kanamatovna Gerbekova and other specialists from the Ryazan' Regional Academic Library who helped us identify V. M. Golovnin's handwriting with a great degree of accuracy. The present study was supported by the Japanese Ministry of Education, Science and Culture, International Scientific Grant-in-Aid Research Program, project "Towards understanding dynamics of language change in Ainu” (2017-2021) (Kiban C 17Ko2743, Principal Investigator: Anna Bugaeva, Tokyo University of Science; Co-investigator: Tomomi Satō).

\section{Abbreviations}

$1,2,3,4 \quad$ person (for 4 th person, see note ap in Table 1 )

A transitive subject

AFF affiliative

ANTIP antipassive

APPL applicative

CL classifier

COP copula

FIN final (particle)

IMP imperative

IMP.POL imperative polite

PL plural

Poss possessive

RECIP reciprocal

REFL reflexive

S intransitive subject

SG singular

VIS.EV visual evidential 


\section{Appendix}

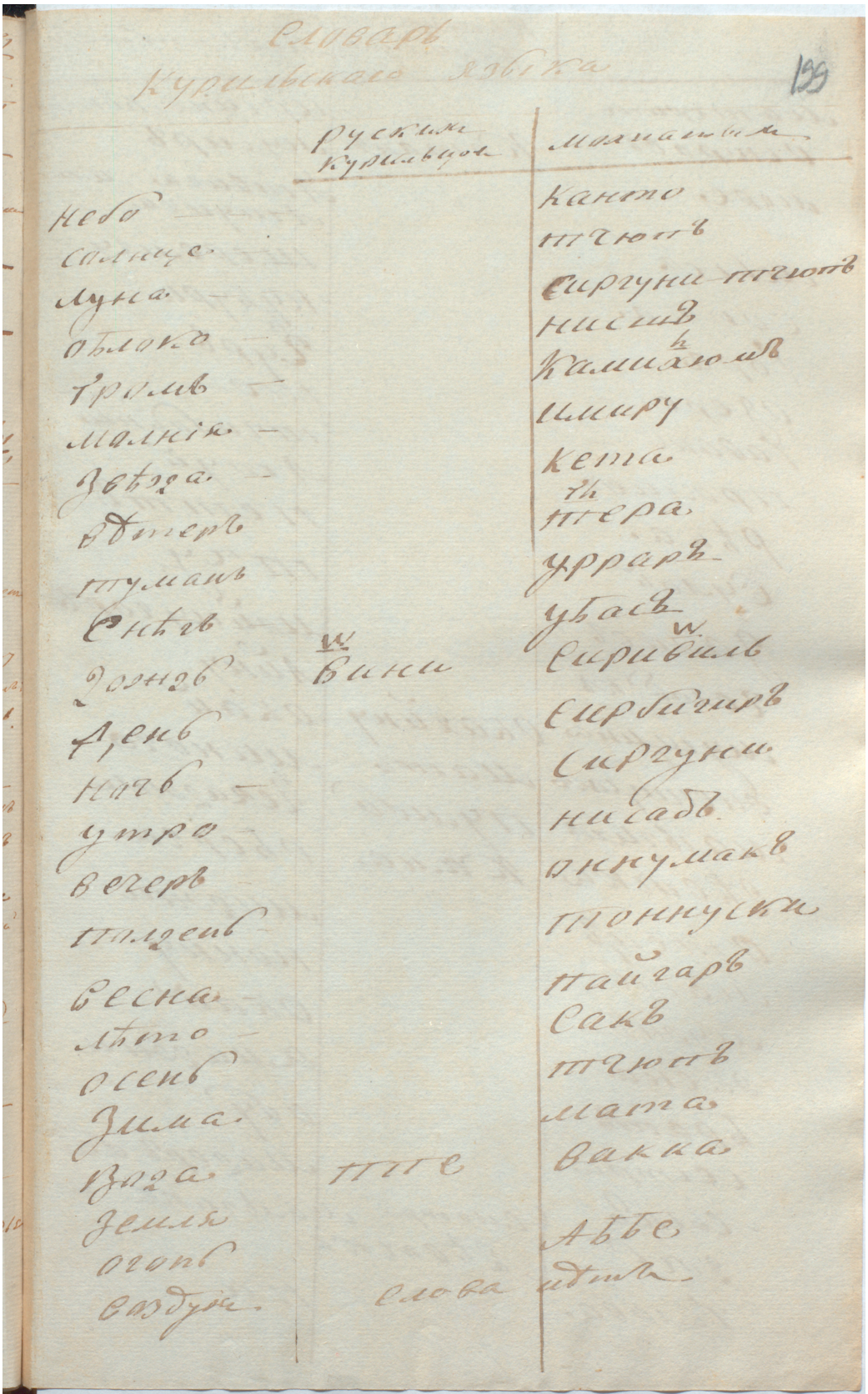

FIGURE 1 Golovnin (1811) (RGAVMF, f. 7, op. 1, \#19, p. 199-203) 


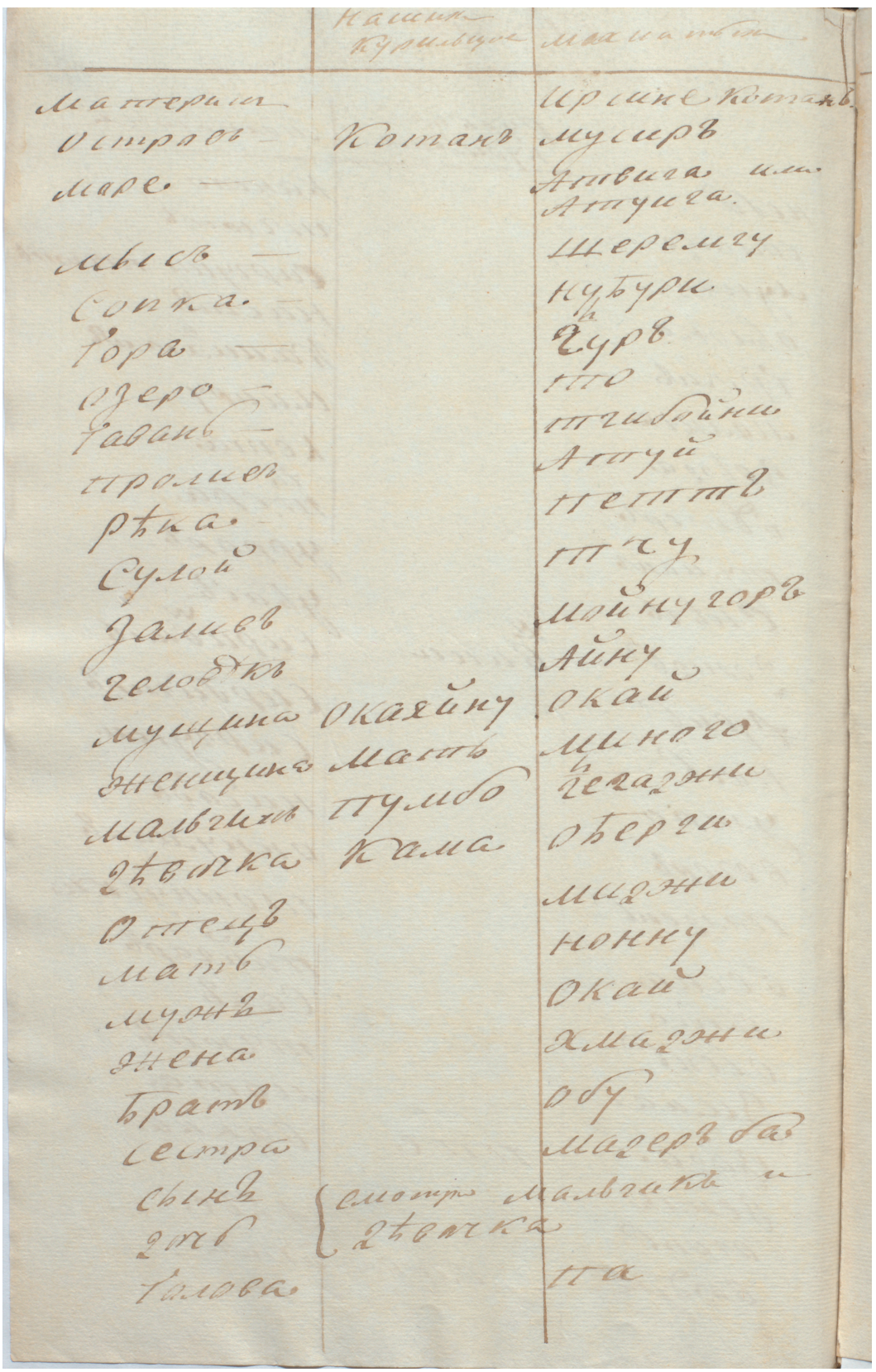




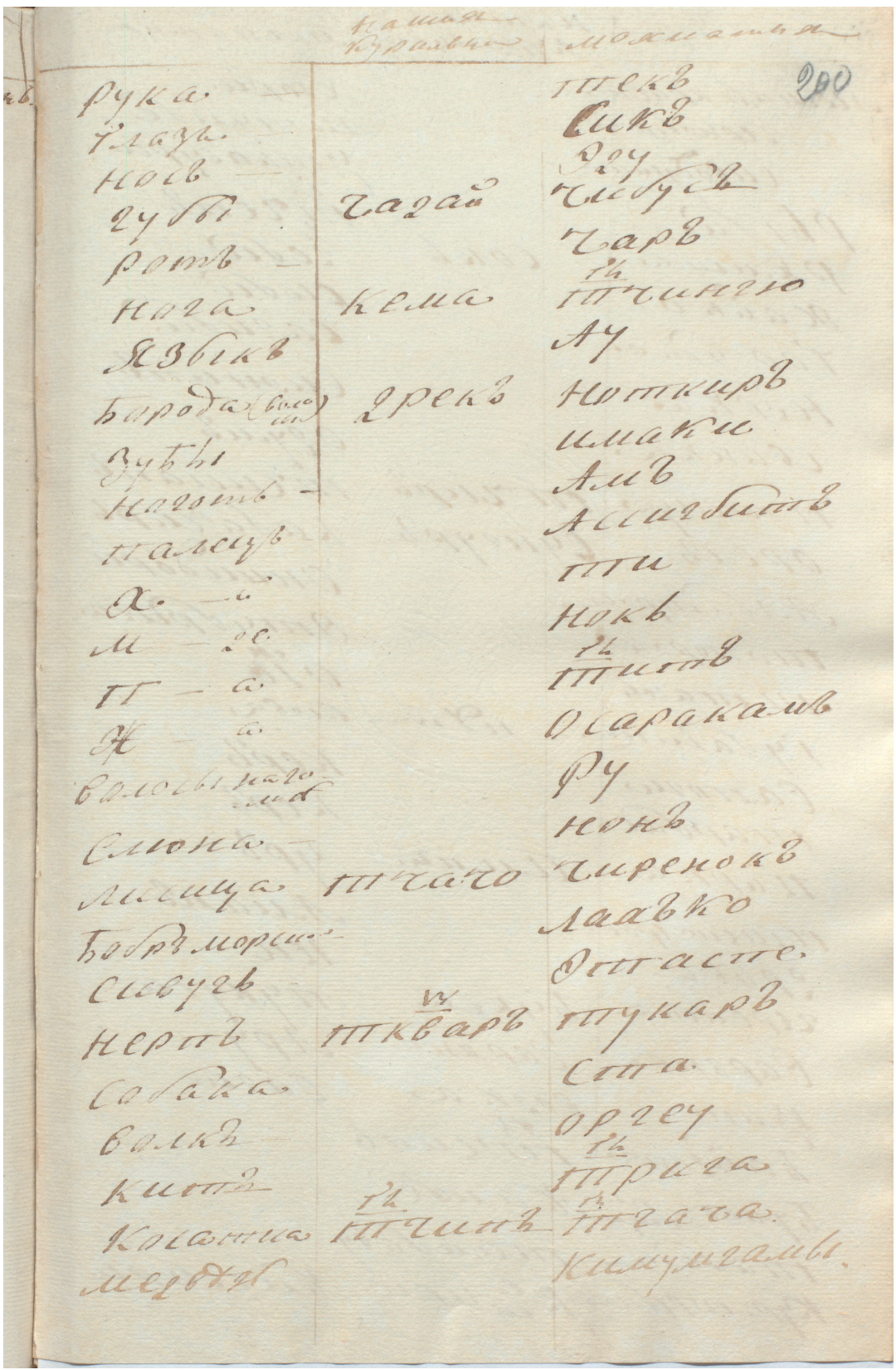




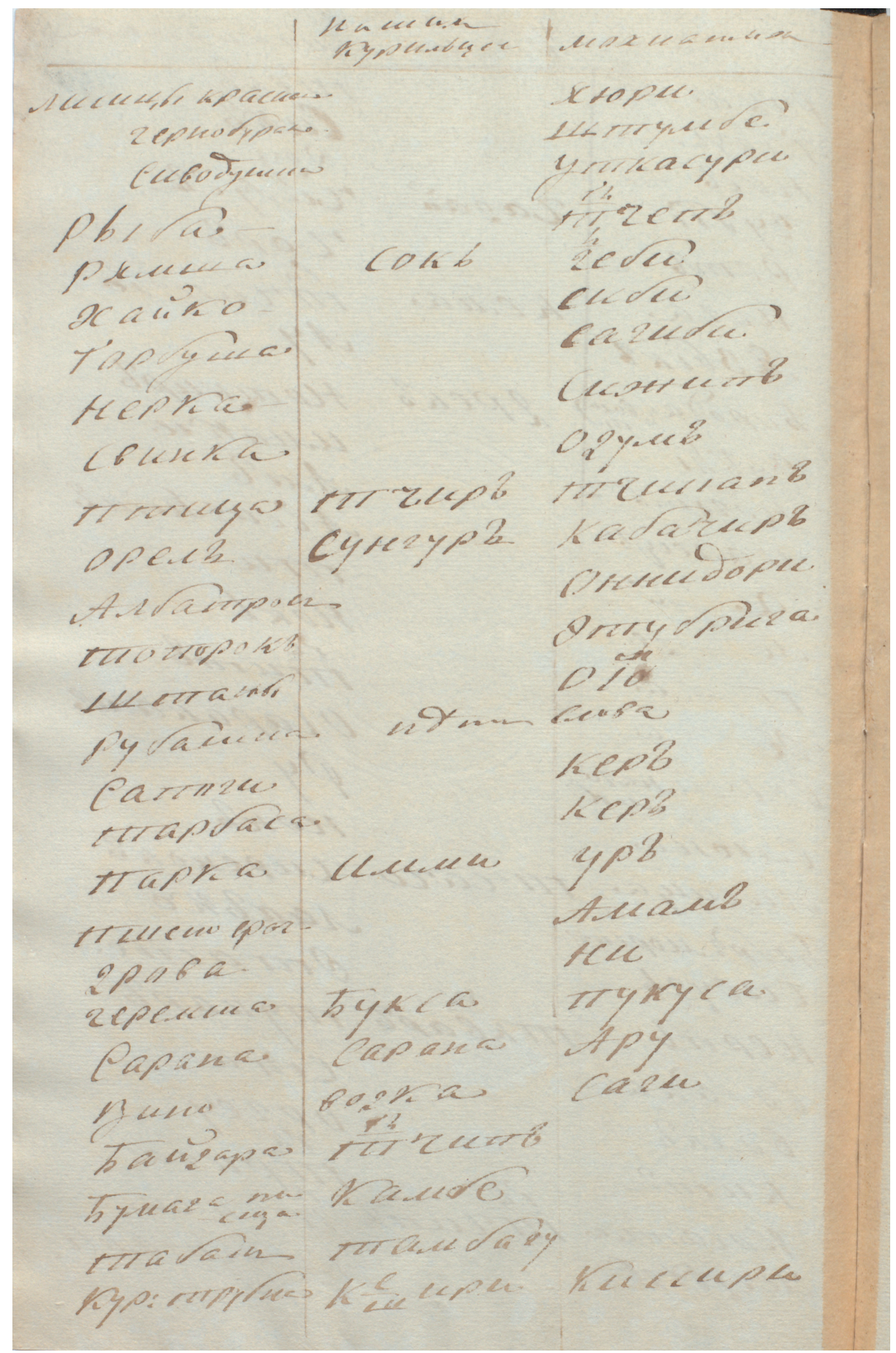




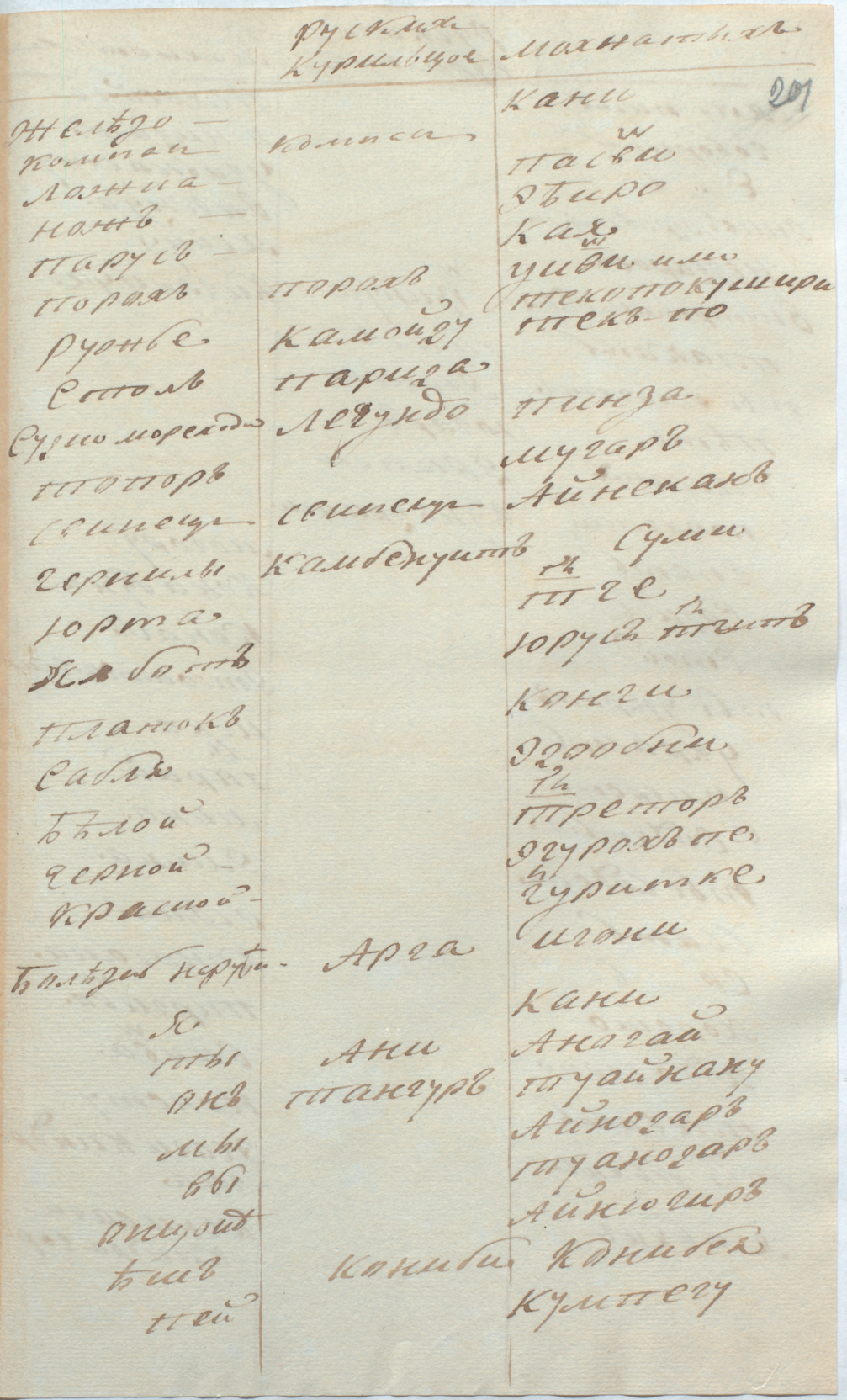




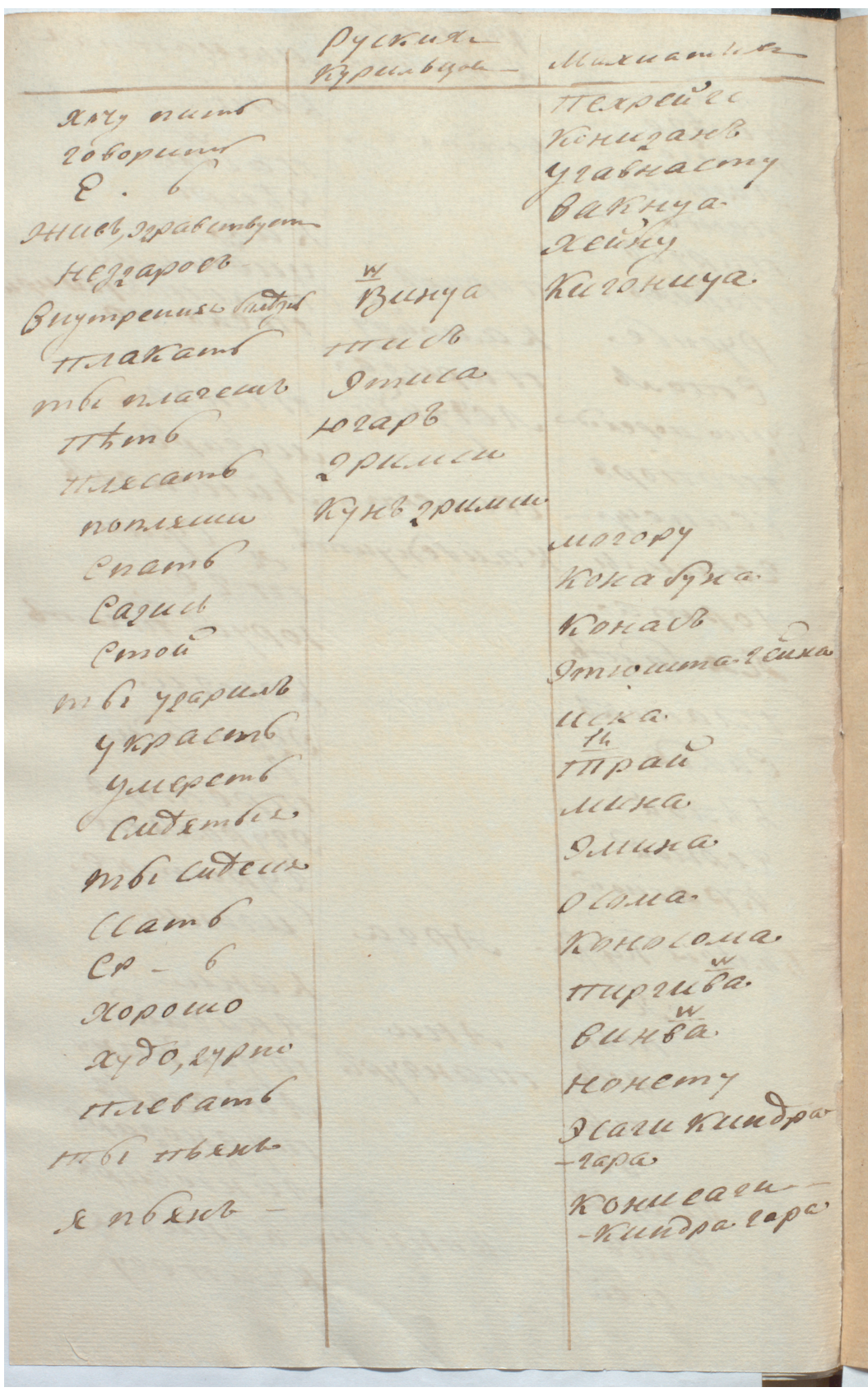




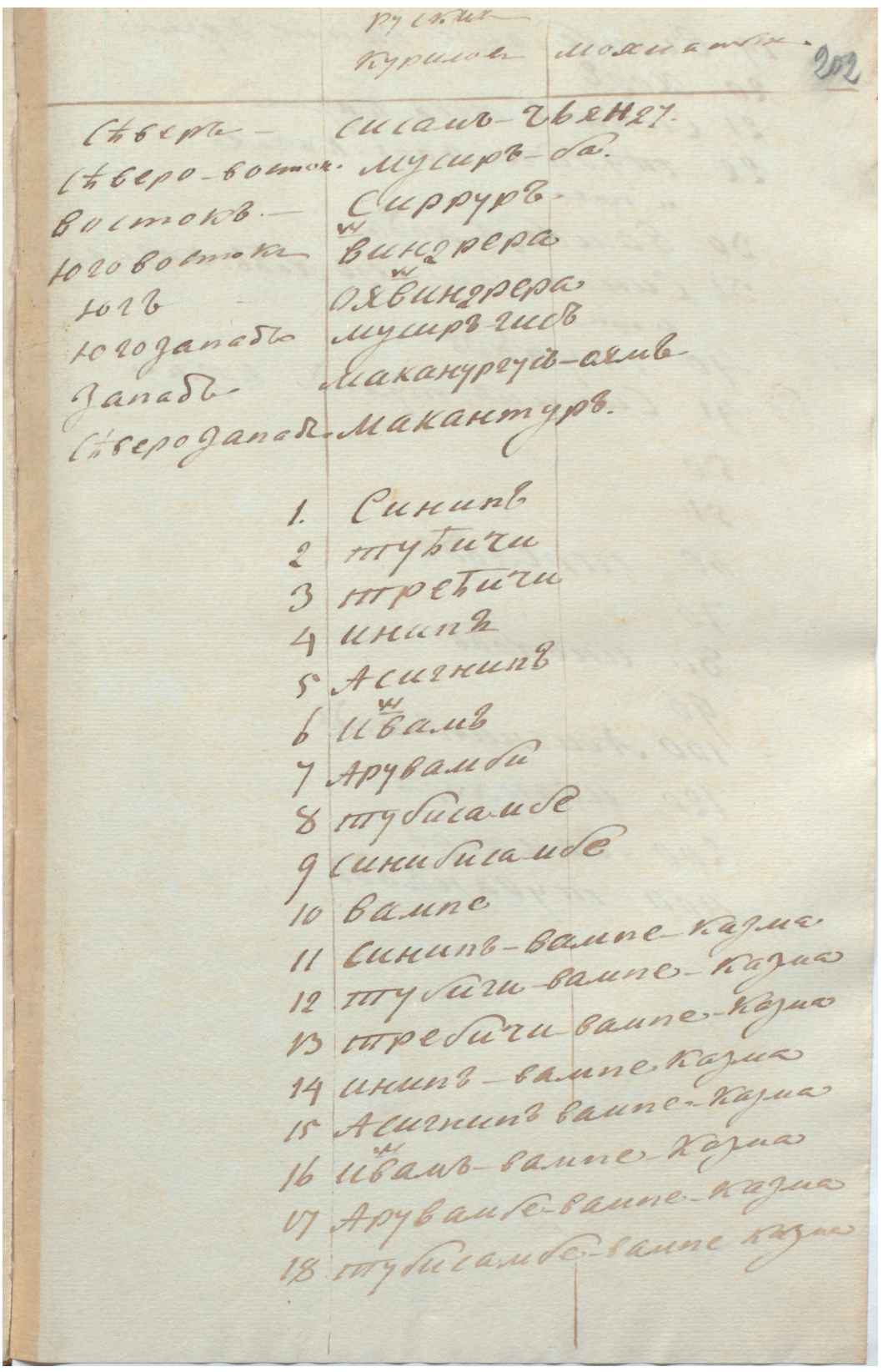




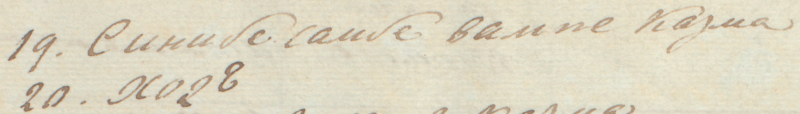

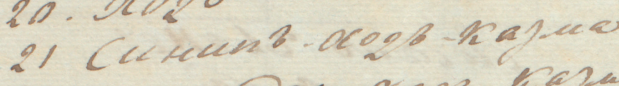

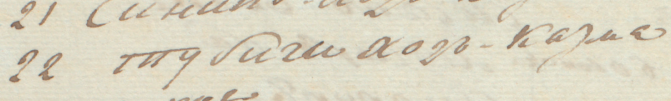

30. Fandegle nd?

1) Curcunb-baudege xodb mata as

40 माप 4008

41. Cubuent-myarde higna

5

$5 \%$

bo mplacod?

70

8)- 14 Herodb

GD 100 accrecuns acodb

1211- LCEसTO2)

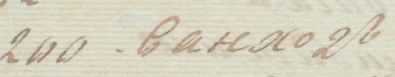

Inll mydatied 


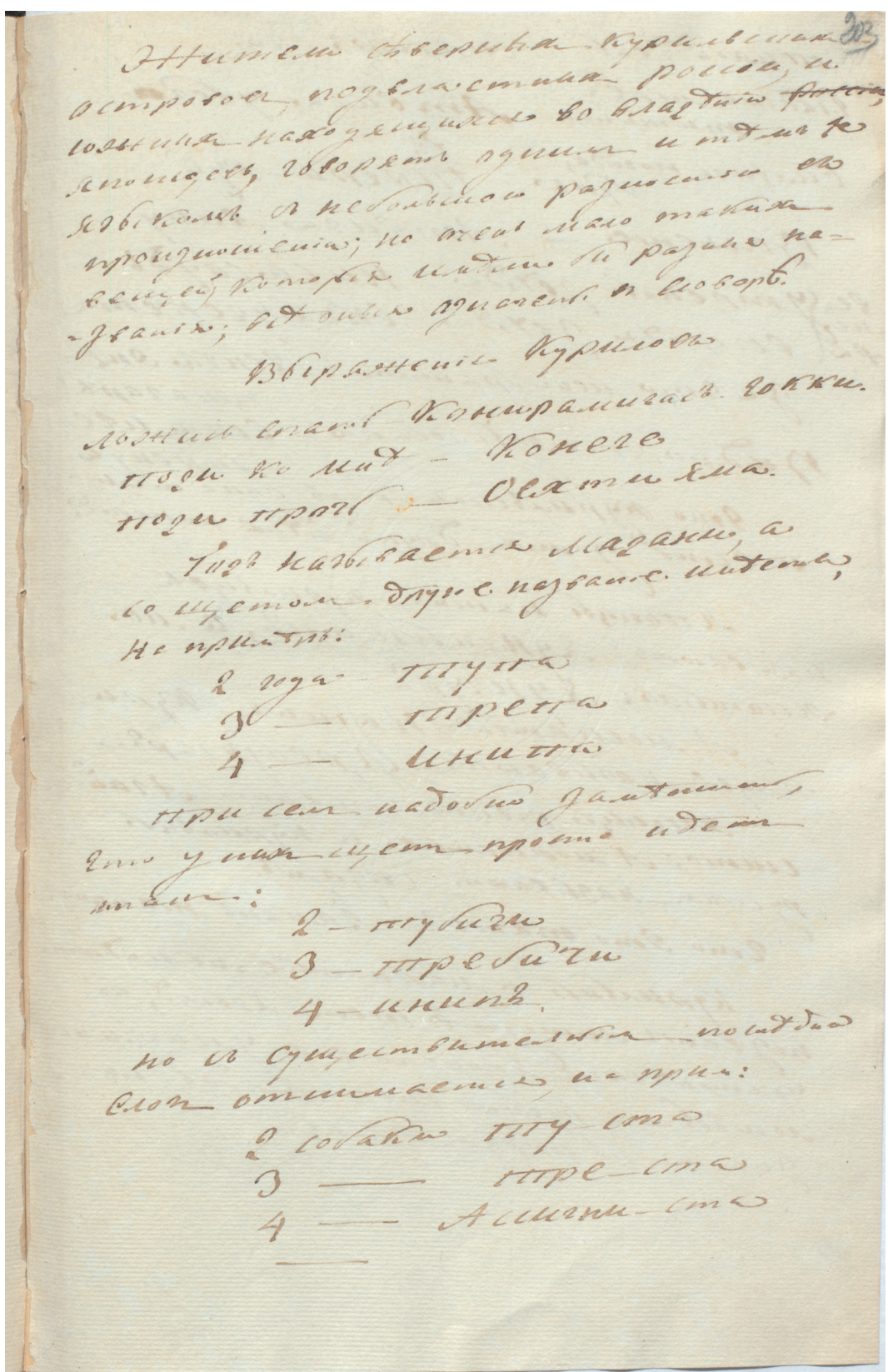




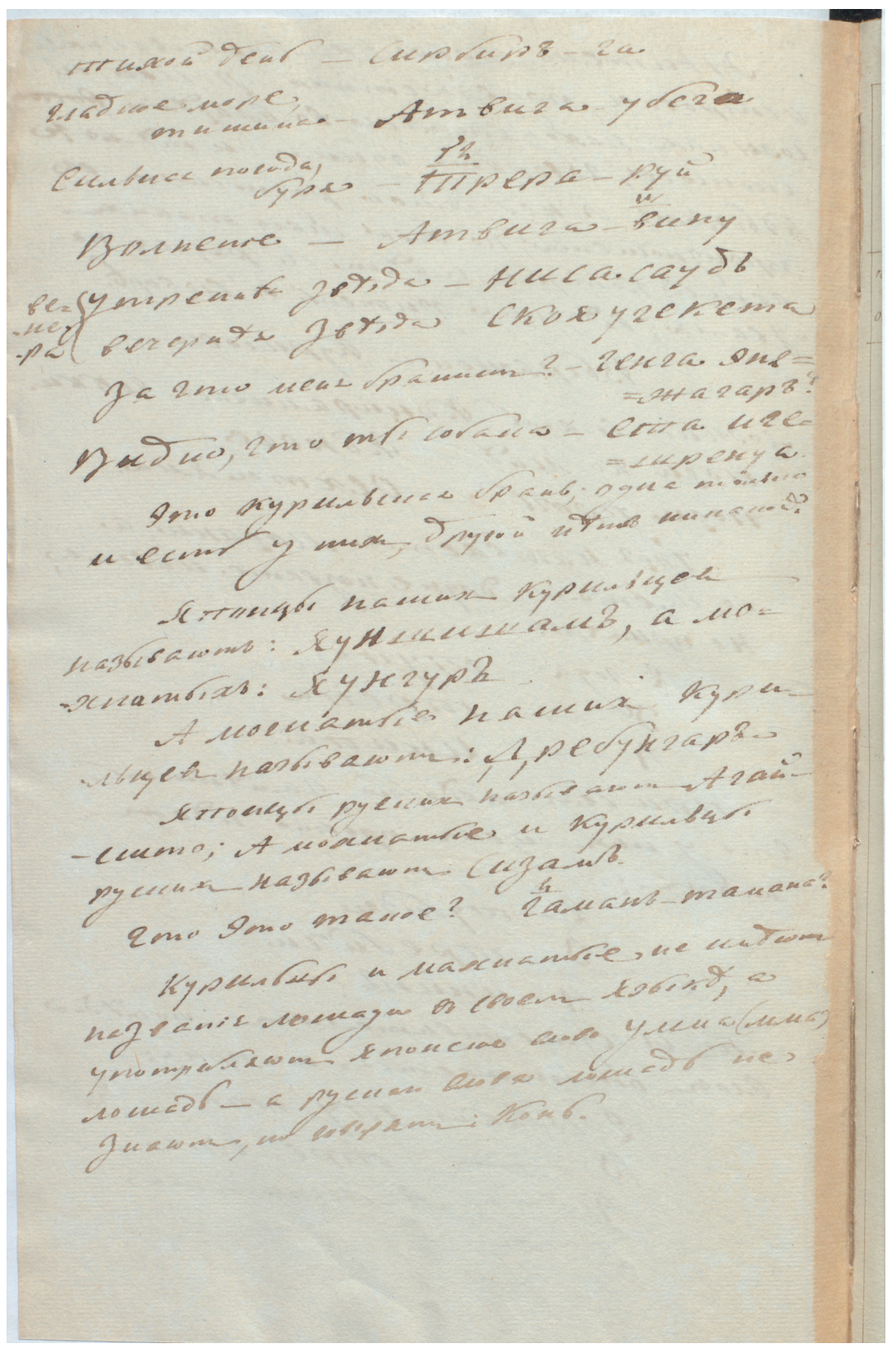




\section{References}

Alonso de la Fuente, José Andrés. 2021. Kuril Ainu Zoonyms and Phytonyms in Pallas's Zoographia and Flora Rossica. International Journal of Eurasian Linguistics 3(1). 1-43.

Batchelor, John. 1938. An Ainu-English-Japanese Dictionary (fourth edition). Tokyo: Iwanami shoten 岩波書店.

Bugaeva, Anna. 2021. Unspecified participant: A case of antipassive in Ainu. In: Katarzyna Janic and Alena Witzlack-Makarevich (eds.). Antipassive Typology, diachrony, and related constructions. Amsterdam and Philadelphia: John Benjamins. 213-246. Dor: https://doi.org/10.1075/tsl.130.o7bug.

Bugaeva, Anna and Anastasiia Gorlova. 2021. An English translation of “Описаніе на первомъ острову живущих куриловъ оихъ обычаяхъ и поведъніи” [A description of the Kurile people living on the first island, their customs and behavior] (1742). Journal of the Center for Northern Humanities (北方人文研究) 14. 135-162.

Chiri 1974 = Chiri Mashiho 知里真志保. 1974 [1936]. アイヌ語法概説 [An outline of Ainu grammar]. In 知里真志保著作集 [The collected works of Chiri Mashiho]. Tōkyō: Heibonsha 平凡社, vol. 4.3-197.

Chiri 1976 = Chiri Mashiho 知里真志保. 1976 [1953]. 知里真志保著作集 (別巻, 1) 分 類アイヌ語辞典。植物変·動物変 [The collected works of Chiri Mashiho. Classified dictionary of the Ainu language: Plants and animals]. Tōkyō: Heibonsha 平凡社 $[394+322$ pp. $]$.

Dybowski 1892 = Radliński, Ignacy. 1892. Słowniki narzeczy ludów kamczackich, I: Słownik narzecza Ainów, zamieszkujących wyspę Szumszu w łańcuchu Kurylskim przy Kamczatce ze zbiorów Prof. B. Dyboskiego [Dictionaries of the languages spoken by the Kamchatkan peoples, I. Dictionary of the language spoken by the Ainu on the islands of Shumshu in the Kuriles near Kamchatka as recorded by Prof. B. Dybowski]. Rozprawy Akademii Umiejętności. Wydziat Filologiczne, Serya II (1). 52-119.

Etorofu omusha moshiwatashi = アトロフコムシヤ申渡. 1821. [An Ainu translation of orders by the Japanese authorities on Etorofu (Iturup) Island]. Sapporo: (unpublished ms. held in) Hokkaidoritsu bunshokan 北海道立文書館.

Golovnin, Vasiliy Mikhajlovich. 1811 (?). Словарь Курильского языка [A Kuril Ainu Glossary]. St. Petersburg: (unpublished ms. held in) The Russian National Archive of the Navy (RGAVMF, f. 7, op. 1, \#19, pp. 199-203).

Golovnin 1816 = Golovnin, Vasiliy Mikhajlovich. 2007[1816]. Zapiski Vasiliya Mikhajlovicha Golovnina v plenu u japontsev v 1811, 1812 i 1813 godakh [Narrative by Vasiliy Mikhajlovich Golovnin in Japanese captivity in 1811, 1812 and 1813]. In:Vasiliy Mikhajlovich Golovnin, Puteshestvija vokrug sveta. Moscow: Drofa. 197-46o.

Golovnin, Vasiliy Mikhajlovich. 1819. Sokraschennye zapiski flota kapitan-lejtenanta Golovnina o plavanii ego na shlyupe Diane, dlya opisi Kuril'skih ostrovov, v 1811 godu 
[Brief notes of the fleet of the captain lieutenant Golovnin about his voyage on the sloop Diana for surveying the Kuril Islands in 1811]. St. Petersburg: Morskaja Tipografija.

Hattori = Hattori Shirō 服部四郎 (ed.). 1964. アイヌ語方言辞典 [Dictionary of Ainu Dialects]. Tōkyō: Iwanami shoten 岩波書店.

Jōdaigo jiten henshū iinkai 1967 = Jōdaigo jiten henshū iinkai 上代語辞典編集委 員会 (ed.). 1967. 時代別国語辞典上代編 [A period-based dictionary of Japanese. Volume: Old Japanese]. Tōkyō: Sanseidō 三省堂.

Kayano Shigeru 萱野茂. 1996. 萱野茂のアイヌ語辞典 [Shigeru Kayano’s Ainu Dictionary]. Tōkyō: Sanseidō 三省堂.

Klaproth, Julius. 1823. Asia polyglotta. Paris: J. M. Eberhart.

Krasheninnikov $1755=$ Krašeninnikovъ, Stepanъ. 1755. Opisanie zemli Kamčatki [Explorations of Kamchatka], 2 vols. Sanktpeterburgъ: pri Imperatorskoj Akademii Naukъ.

Kubodera 1992 = Kubodera Itsuhiko 久保寺逸彦. 1992. アイ邓語・日本語辞典: 久保 寺逸彦アイヌ語収録ノー卜調查報告書 [An Ainu-Japanese dictionary: A report on Itsuhiko Kubodera's Ainu notes]. Sapporo: Iwanami shinsho 岩波新書.

Mabuchi 1957 = Mabuchi, Kazuo 馬淵和夫. 1957. 古事記のシ・オ・ホのかな [Kana symbols shi, o, and ho in Kojiki]. 国語学 [Japanese language studies] 31. 12-31.

Ministry of Culture and Tourism of Ryazan' Region, the Ryazan' Regional Academic Library (ed.). 2021. Biblioteka Golovninykh. Katalog [The Golovnins' library. A catalogue]. Ryazan': G BU к Rо "Biblioteka im. Gor'kogo".

Murayama Shichirō 村山七郎. 1971. 北千島アイヌ語・文献学的研究 [The Kuril Ainu language. A Study on Sources]. Tōkyō: Yoshikawa kōbunkan 吉川弘文館.

Nakagawa = Nakagawa, Hiroshi 中川裕. 1995. アイヌ語千歳方言辞典 [A dictionary of the Chitose dialect of Ainu]. Tōkyō: Sōfūkan 草風館.

Nakagawa, Hiroshi and Mika Fukazawa. [to appear]. Hokkaido Ainu dialects: towards a classification of Ainu dialects. In Anna Bugaeva (ed.). Handbook of the Ainu Language (HJLL V.12). Berlin/Boston: De Gruyter Mouton.

Narita and Kindaichi $1972=$ Moshiogusa 藻汐草. In Narita Shūichi 成田修一 (ed.). ア イ語資料草書・金田一京助解說 [Ainu primary sources, with commentary by Kindaichi Kyōsuke]. Tōkyō: Kokusho kankōkai 国書刊行会.

Nihon daijiten kankōkai 日本大辞典刊行会. 1973. 日本国語大辞典. [A dictionary of the Japanese language]. Tokyo: Shōgakukan 小学館.

Sasaki Akihisa and Tanimoto Toshikazu (eds.). 佐々木利和・谷本晃久(編). 2019. 蝦夷 語集索引 [An index of Ezogoshū]. Sapporo: Center for Ainu and Indigenous Studies Hokkaido University 北海道大学アイヌ先住民センター.

Satō Tomomi 佐藤知已. 1995. 蝦夷言いろは引の研究 [A study of Ezokotoba irohabiki]. Sapporo: Faculty of Letters, Hokkaido University 北海道大学文学部. 
Satō and Bugaeva 2019 = Sato Tomomi and Anna Bugaeva. 2019. The Study of Old Documents of Hokkaido and Kuril Ainu: Promise and Challenges. Northern Language Studies (北方言語研究) 9.67-93.

Tamura Suzuko 田村すず子. 1996. アイヌ語沙流方言辞典 [A Dictionary of Saru Ainu]. Tōkyō: Sōfūkan 草風館.

Torii Ryūzō 鳥居竜蔵. 1903. 千島アイヌ [The Kuril Ainu]. Tōkyō: Yoshikawa kōbunkan 吉川弘文館.

Vovin, Alexander. 1993. A reconstruction of Proto-Ainu. Leiden: E. J. Brill.

Voznesensky = Voznesensky, Ilya Gavrilovich. ca. 1844. Kuril'skie Slova [Kuril words]. St. Petersburg: (unpublished ms. held in the St. Petersburg) Branch of the Archive of the Russian Academy of Sciences (f.53, op.1, \#43).

Walker, Brett L. 2007. Mamiya Rinzō and the Japanese exploration of Sakhalin Island: cartography and empire. Journal of Historical Geography 33 (2). 283-313.

Zajac = Zajac, Malgorzata ザヨンツ・マウゴジャータ. 20o9. 千島アイヌの軌跡 $[$ A history of Kuril Ainu]. Tōkyō: Sōfūkan 草風館. 